\title{
A systematic review on literature-based discovery workflow
}

\author{
Menasha Thilakaratne ${ }^{\text {Corresp., } 1}$, Katrina Falkner ${ }^{1}$, Thushari Atapattu ${ }^{1}$ \\ ${ }^{1}$ Faculty of Engineering, Computer and Mathematical Sciences, The University of Adelaide, Adelaide, South Australia, Australia \\ Corresponding Author: Menasha Thilakaratne \\ Email address: menasha.thilakaratne@adelaide.edu.au
}

As scientific publication rates increase, knowledge acquisition and the research development process have become more complex and time-consuming. Literature-Based Discovery ( $L B D)$, supporting automated knowledge discovery, helps facilitate this process by eliciting novel knowledge by analysing existing scientific literature. This systematic review provides a comprehensive overview of the LBD workflow by answering nine research questions related to the major components of the LBD workflow (i.e. input, process, output, and evaluation). With regards to the input component, we discuss the data types and data sources used in the literature. The process component presents filtering techniques, ranking/thresholding techniques, domains, generalisability levels, and resources. Subsequently, the output component focuses on the visualisation techniques used in LBD discipline. As for the evaluation component, we outline the evaluation techniques, their generalisability, and the quantitative measures used to validate results. To conclude, we summarise the findings of the review for each component by highlighting the possible future research directions. 


\title{
A Systematic Review on Literature-Based Discovery Workflow
}

\author{
Menasha Thilakaratne ${ }^{1}$, Katrina Falkner ${ }^{1}$, and Thushari Atapattu ${ }^{1}$ \\ ${ }^{1}$ Faculty of Engineering, Computer and Mathematical Sciences, The University of \\ Adelaide, Adelaide, South Australia, Australia \\ Corresponding author: \\ Menasha Thilakaratne ${ }^{1}$ \\ Email address: menasha.thilakaratne@adelaide.edu.au
}

\begin{abstract}
As scientific publication rates increase, knowledge acquisition and the research development process have become more complex and time-consuming. Literature-Based Discovery (LBD), supporting automated knowledge discovery, helps facilitate this process by eliciting novel knowledge by analysing existing scientific literature. This systematic review provides a comprehensive overview of the LBD workflow by answering eight research questions related to the major components of the LBD workflow (i.e. input, process, output, and evaluation). With regards to the 'input' component, we discuss the data types and data sources used in the literature. The 'process' component presents filtering techniques, ranking/thresholding techniques, and LBD resources. Subsequently, the 'output' component focuses on the visualisation techniques used in LBD discipline. As for the 'evaluation' component, we outline the evaluation techniques, their generalisability, and the quantitative measures used to validate results. To conclude, we summarise the findings of the review for each component by highlighting the possible future research directions.
\end{abstract}

\section{INTRODUCTION}

Due to the exponential growth of scientific publications, keeping track of all research advances in the scientific literature has become almost impossible for a scientist (Cheadle et al., 2017). As a result, scientific literature has become fragmented and individual scientists tend to deal with fragments of knowledge based on their specialisation. Consequently, valuable implicit associations that connect these knowledge fragments tend to remain unnoticed since scientists in each specialisation have only seen part of the big picture. Literature-Based Discovery $(L B D)$ supports cross-disciplinary knowledge discovery to elicit these hidden associations to recommend new scientific knowledge. The recommended novel associations can greatly assist scientists in formulating and evaluating novel research hypotheses (Ganiz et al., 2005). While reducing the time and effort, this will also promote scientists to discover new areas of research.

\section{Brief History}

LBD was developed as a research field from the medical discoveries published by Swanson since 1986. In his first discovery, he manually analysed the titles of two literature; Fish oil and Raynaud's disease (Swanson, 1986). Swanson has observed that the patients with Raynaud tend to have high blood viscosity and high platelet aggregation. He has also noted that Fish Oil contains EPA (eicosapentaenoic acid) that helps to decrease the blood viscosity and platelet aggregation. By combining these knowledge pairs, he generated the hypothesis; "Raynaud can be cured using Fish Oil". Furthermore, he also observed that the two literature he was referring are disjoint, i.e. the articles in the two literature sets have not mentioned, cited or co-cited each other. Consequently, he published these findings that were deduced using the ABC model (see Discovery Models section). His second discovery followed the same process where he manually examined the titles of Migraine and Magnesium to detect implicit associations that connects the two literature (Swanson, 1988). Later, his observations were proven through laboratory experiments that demonstrate the validity of his thinking process (Ramadan et al., 1989). 
Even though the early work of Swanson was mostly performed manually by merely analysing the article titles and their word co-occurrence frequencies, they formed the foundation of the field. In accordance with Swanson's experiments, the existing disperse knowledge fragments in literature can be accumulated in such a way to develop novel semantic relationships that have not drawn any awareness before (a.k.a undiscovered public knowledge) (Swanson, 1986). These connectable disperse knowledge fragments in the literature may exist as; 1) hidden refutations or qualifications, 2) undrawn conclusion from different knowledge branches, 3) cumulative weak tests, 4) analogous problems, and/or 5) hidden correlations (Davies, 1989). In a later study, Swanson also pointed out the importance of studying cases where the interaction of the two literature sets is not null (i.e. the literature sets are not disjoint), but populated by few articles (a.k.a literature-based resurrection (Swanson, 2011), scientific arbitrage (Smalheiser, 2012a)).

\section{Discovery Models}

Most of the LBD literature is based on the fundamental premise introduced by Swanson, namely $A B C$ model (Swanson, 1986). It employs a simple syllogism to identify the potential knowledge associations (a.k.a. transitive inference). That is, given two concepts $A$ and $C$ in two disjoint scientific literature, if concept $A$ is associated with concept $B$, and the same concept $B$ is associated with concept $C$, the model deduces that the concept $A$ is associated with the concept $C$. The popular ABC model has two variants named as open discovery and closed discovery.

Open discovery is generally used when there is a single problem with limited knowledge about what concepts can be involved. The process starts with an initial concept related to the selected research question/problem (A-concept). Afterwards, the LBD process seeks the relevant concepts that ultimately lead to implicit associations (C-concepts). In other words, only the concept A is known in advance and concepts B and C are identified by the LBD process. Therefore, this model can be viewed as a knowledge discovery process that assists to generate novel research hypotheses by examining the existing literature. Unlike the open discovery process, closed discovery model attempts to discover novel implicit associations between the initially mentioned A-concept and C-concept (a.k.a concept bridges). Thus it represents hypotheses testing and validation process. More explicitly, the LBD process starts with user-defined A-concept and C-concept and the output will be the intermediate B-concepts that represents the associations between the two user-defined domains.

Even though the prevalent $\mathrm{ABC}$ model have contributed in numerous ways to detect new knowledge, it is merely one of several different types of discovery models that facilitates LBD process. In this regard, Smalheiser (2012a) points out the importance of thinking beyond the ABC formulation and experimenting alternative discovery models in the discipline. Despite the simplicity and power of the ABC model, it also suffers from several limitations such as the sheer number of intermediate terms that exponentially expands the search space and producing a large number of target terms that are hard to interpret manually (Smalheiser, 2012a). Even though the research in LBD have suggested various ways to overcome the aforementioned two limitations, most of these studies rely on similarity based measures to rank the target terms. This will result in LBD systems that merely detect incremental discoveries. In addition, the field requires to explore various interestingness measures that allows to customise the LBD output that cater different types of scientific investigations (Smalheiser, 2012a).

With respect to other LBD discovery models that are enhanced based on ABC discovery structure include $A n C$ model where $\mathrm{n}=(B 1, \ldots, B n)$ (Wilkowski et al., 2011), combination of both open and closed discovery models (Petriě et al., 2009), context-based $A B C$ model (Kim and Song, 2019), and contextassignment-based ABC model (Kim and Song, 2019). Moreover, recent studies have attempted to further explore alternative discovery models that deviate from the typical ABC discovery setting. These new directions include storytelling methodologies (Sebastian et al., 2017b), analogy mining (Mower et al., 2016), outlier detection (Gubiani et al., 2017), gaps characterisation (Peng et al., 2017), and negative consensus analysis (Smalheiser and Gomes, 2015). For a comprehensive discussion of contemporary discovery models and future directions, please refer (Smalheiser, 2017, 2012a).

\section{Purpose of the Review}

Even though there are several review papers (Gopalakrishnan et al., 2019; Henry and McInnes, 2017; Sebastian et al., 2017a; Ahmed, 2016) published on LBD, the field still lacks systematic literature reviews. Therefore, the existing reviews merely cover a subset of LBD literature and do not provide a comprehensive classification of the LBD discipline. To address this gap, we present a large-scale 


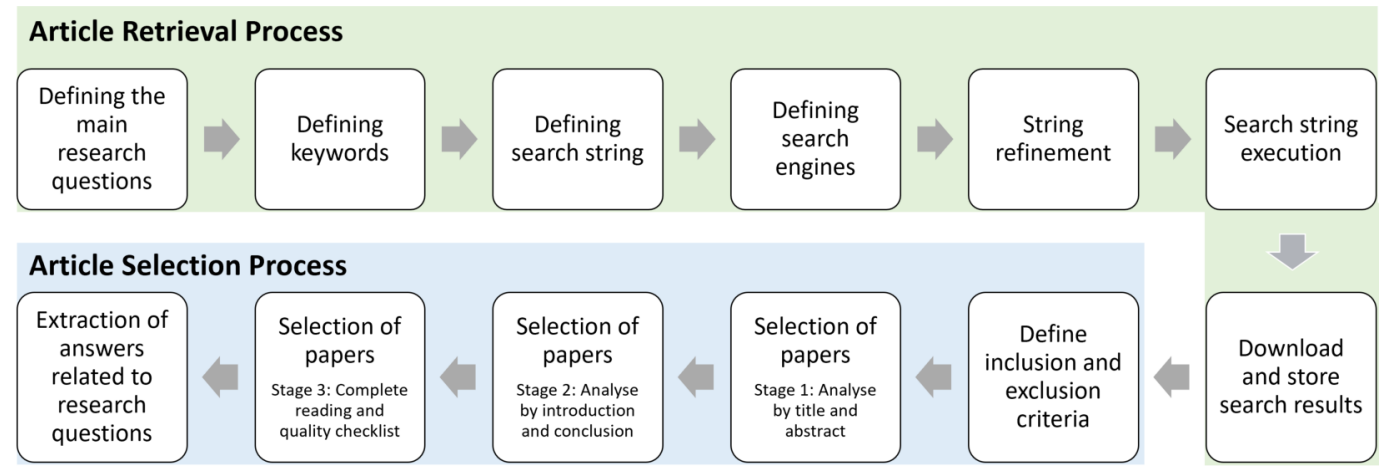

Figure 1. Process of the systematic literature review

systematic review by analysing 176 papers that were selected by manually analysing 475 papers. On the contrary to the existing traditional reviews, systematic reviews follow a rigorous and transparent approach to ensure the future replicability of results through the use of a clear systematic review protocol, and to minimise the bias in results by focusing on empirical evidence to present results, not preconceived knowledge (Mallett et al., 2012).

Another persistence research deficiency of other literature reviews is due to their limited and ad-hoc focus points. To date, none of the existing reviews focuses on the LBD workflow as a whole. Moreover, despite the importance of LBD components such as input, output, and evaluation, the existing reviews have not paid attention to critically analyse the state-of-the-art and the limitations of these components. To overcome these two limitations, in this review we provide a sequential walk through of the entire LBD workflow by providing new insights into the LBD components such as input, output, and evaluation.

Furthermore, we have also observed that most of the existing reviews have restricted their scope only to medical-related LBD studies. Consequently, these reviews are lacking the discussions of LBD in non-medical and domain independent setting. To cater this issue, we have examined the LBD literature in both medical and non-medical domains in this review.

More specifically, our contributions are; 1) being the first systematic literature review that covers every component of the LBD workflow, 2) shedding light on components in LBD workflow such as input, output, and evaluation that have not been critically analysed or categorised by the existing reviews, 3) answer each of our research questions using novel, up-to-date and comprehensive categorisations compared to the existing reviews, and 4) critiquing LBD literature independently from domain without restricting to only medical-related LBD studies.

\section{METHODS}

The overall process of this systematic review adheres the steps of Systematic Literature Reviews in Computer Science (Weidt and Silva, 2016) as illustrated in Figure 1.

\section{Article Retrieval Process}

We used six keywords and six databases to retrieve the articles for this review. Each keyword is searched in the title, abstract or keywords depending on the search options given by the databases. To ensure that we have not missed any useful articles, we also added the full reference list of a latest LBD review (Henry and McInnes, 2017). The article retrieval process with relevant statistics is summarised in Table 1.

\section{Article Selection Process}

We only included journals and conference proceedings that are in the English language in our analysis. We excluded other types of articles such as reviews, books, book chapters, papers reporting lessons learned, keynotes, and editorials. We also eliminated the papers that provide the theoretical perspective of LBD as our research questions are focused to assess the LBD discipline in terms of computational techniques. We also excluded articles of page count 4 or below as such articles mainly contain researchin-progress. The entire article selection of this review was performed in three stages (Weidt and Silva, 2016); Stage 1: analysing only title and abstract, Stage 2: analysing introduction and conclusion, and 
Table 1. Statistics of the article retrieval process

\begin{tabular}{|l|l|l|l|l|l|l|l|}
\hline Keyword & $\begin{array}{l}\text { Web of } \\
\text { Science }\end{array}$ & Scopus & PubMed & $\begin{array}{l}\text { ACM Digital } \\
\text { Library }\end{array}$ & $\begin{array}{l}\text { IEEE } \\
\text { Xplore }\end{array}$ & $\begin{array}{l}\text { Springer } \\
\text {-Link }\end{array}$ & $\begin{array}{l}\text { Total } \\
\text { count }\end{array}$ \\
\hline Query $1^{a}$ & 161 & 68 & 75 & 15 & 15 & 8 & 342 \\
\hline Query $2^{b}$ & 14 & 0 & 4 & 1 & 2 & 1 & 22 \\
\hline Query $3^{c}$ & 14 & 0 & 0 & 0 & 0 & 1 & 15 \\
\hline References from Henry and McInnes (2017) \\
Total Article count \\
\hline
\end{tabular}

a "literature based discovery" OR "literature based discoveries"

b "literature based knowledge discovery" OR "literature based knowledge discoveries"

c "literature related discovery" OR "literature related discoveries"

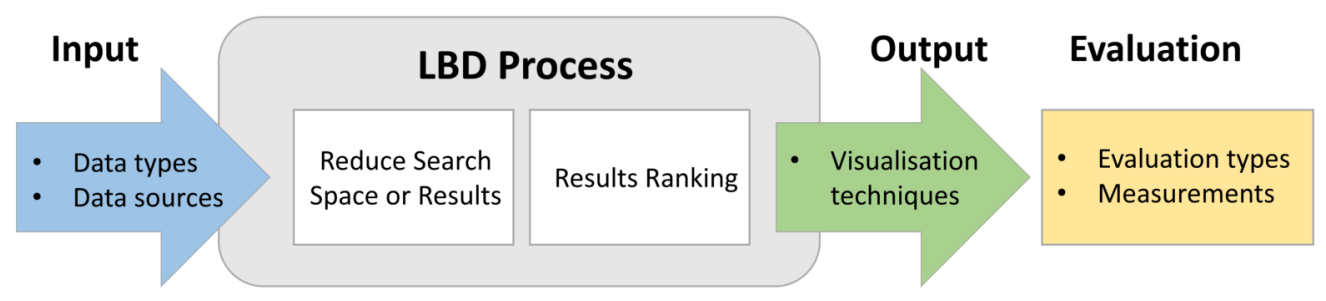

Figure 2. Main components of the LBD workflow

Stage 3: read complete article and quality checklist. In total, we obtained 176 papers for this review (listed in https://tinyurl.com/selected-LBD-articles).

\section{REVIEW OVERVIEW}

In this review we seek answers for 8 research questions that are grouped into four categories by considering the workflow of LBD process as illustrated in Figure 2.

\section{Input Component}

What are the data types considered for knowledge discovery?

What are the data sources used in LBD research?

\section{Process Component}

What are the filtering techniques used in the LBD process?

What are the ranking/thresholding mechanisms used in LBD literature?

What are the domain independent and domain dependent resources utilised in LBD research?

\section{Output Component}

What are the visualisation techniques used to display the results in LBD research?

\section{Evaluation Component}

What are the LBD evaluation types and their domain dependencies?

What are the quantitative measurements used to assess the effectiveness of the results?

To increase the readability of our review, we have cited a limited number of literature for each research question. However, a complete list of references that supports the proposed categorisations and conclusions of the research questions are listed in https://tinyurl.com/full-references. 


\section{INPUT COMPONENT}

This section analyses the input component of the LBD workflow to get an overview of the data structures and databases used in the literature.

\section{What are the data types considered for knowledge discovery?}

LBD literature makes use of different data types as their input of the knowledge discovery process. The selection of the most suitable data type is one of the key design decisions, as they should represent the most important entities and relationships of the article to perform an efficient knowledge discovery. The data types used in the LBD literature can be categorised as follows.

Title only: Some LBD studies (Swanson and Smalheiser, 1997; Cherdioui and Boubekeur, 2013) have only considered the article title as the input of the knowledge discovery process. This input type selection might have influenced from Swanson's initial work as he only utilised the titles to uncover the hidden associations (e.g., Raynaud $\leftrightarrow$ Fish Oil). Even though the article title contains limited information, Sebastian et al. (2017b) have reported that using only titles for knowledge discovery tend to produce better results compared to analysing abstracts.

Title and Abstract: The most common data type selection in literature is using both title and abstract (Lever et al., 2017; Sebastian et al., 2017b). The main reasons for this selection over full-text analysis could be; 1) Reducing noise: Typically the title and abstract include the most important concepts that best describe the study than considering the full-text, 2) Data retrieval constraints: Most APIs of the literature databases only support metadata retrieval, and 3) Reducing computational complexity: As the content of title and abstract is restricted the time and space complexities are reduced compared to full-text analysis.

Full-text: Few studies (Lever et al., 2017; Vicente-Gomila, 2014) have considered the entire content of articles as their input type. It has been reported that using full-text yields better results over title and abstract analysis. (Seki and Mostafa, 2009). However, it is also important to pay attention as to what sections of the full-text need to be analysed to obtain better results. For instance, does analysing only the methodological-related sections of the article produce better results than analysing the entire article? Such sections-related analysis have not been preformed in LBD literature yet.

Selected articles only: While most of the studies have used data retrieved from literature database search engines (e.g., Medline) for analysis, Cameron et al. (2015) have only considered the reference lists of Swanson's LBD publications. Considering only the 65 articles cited in Swanson's Raynaud $\leftrightarrow$ Fish Oil LBD paper (Swanson, 1986) as the input of the knowledge discovery process can be taken as an example. However, since these reference lists are manually analysed and selected, whether this data type selection reflects the complexity of the real world data is doubtful.

Entire literature database: Several research studies (Lever et al., 2017; Yang et al., 2017) have considered the entire literature database as the LBD input without only limiting to articles retrieved for a given query (e.g., subset of the articles retrived for the query 'Fish oil'). Since the primary focus of LBD research is in the medical domain, the literature database that has been mainly considered for analysis is Medline. Additionally, other sources such as SemMedDB (Cohen et al., 2014) and PubMed Central Open Access Subset articles (Lever et al., 2017) have also been considered as the input.

Keywords: Some research approaches have employed the keywords of the articles as the input data type (Pusala et al., 2017; Hu et al., 2010). The mostly utilised keyword type is Medical Subject Headings $(\mathrm{MeSH})$ that are associated with Medline records. It is considered that MeSH descriptors are accurate and medically relevant as National Library of Medicine (NLM) employs trained indexers to assign them to the Medline articles. Therefore, it is considered as a reliable source of representing the content of the article.

Other metadata: Few studies have analysed other metadata of the research articles such as author details (Sebastian et al., 2017b), publisher details (Sebastian et al., 2015) and reference details (Kostoff et al., 2008a) to glean additional clues for the possible links in the knowledge discovery process. The results prove that such metadata enhances the predictability of implicit knowledge associations (Sebastian et al., 2017b). 
Other traditional data types: While the majority of the studies have focused only on the analysis of research papers, some approaches have been conducted using other traditional data types such as patents (Vicente-Gomila, 2014; Maciel et al., 2011), and TREC MedTrack collection of clinical patient records (Symonds et al., 2014), case reports (Smalheiser et al., 2015) as their input to the LBD process.

Non-traditional data types: Few research studies have attempted to perform LBD using non-traditional data types such as tweets (Bhattacharya and Srinivasan, 2012), Food and Drug Administration (FDA) drug labels (Bisgin et al., 2011), Popular Medical Literature (PML) news articles (Maclean and Seltzer, 2011), web content (Gordon et al., 2002), crime incident reports (Schroeder et al., 2007) and commission reports (Jha and Jin, 2016a). Their results have proved the suitability of LBD discovery setting in a non-traditional context to elicit hidden links.

The Data unit of analysis denotes the types of data extracted from the above-discussed data types to represent the knowledge associations. Since most of the LBD research performed in medicine, the most common term representation is using $U M L S$ and MeSH (Lever et al., 2017; Preiss and Stevenson, 2017). Apart from these two medical resources, other medical databases such as Entrez Gene (Kim et al., 2016), HUGO (Petric et al., 2014), LocusLink (Hristovski et al., 2005), OMIM (Hristovski et al., 2003) and PharmGKB (Kim and Park, 2016) have also being used extract data units. LBD studies in other domains mainly consider word or word phrases (n-grams) as their term representation (Qi and Ohsawa, 2016) that have been extracted using techniques such as Part-Of-Speech (POS) tag patterns.

\section{What are the data sources used in LBD research?}

Medline/PubMed is extensively being used as the main data source of the LBD literature (Lever et al., 2017). Additionally, other data sources such as PubMed Central (PMC) Open Access (Ding et al., 2013), Science Direct (Vicente-Gomila, 2014), Web of Science (Sebastian et al., 2015), IEEE Xplore Digital Library (Qi and Ohsawa, 2016), Engineering Village (Kibwami and Tutesigensi, 2014), ProQuest (Kibwami and Tutesigensi, 2014), EBSCO Host (Kibwami and Tutesigensi, 2014), INSPEC (Ye et al., 2010) have also been employed by several other LBD approaches to retrieve the articles for analysis.

The patent-based LBD studies (Vicente-Gomila, 2014), have considered patent databases such as Thomson Innovation, United State Patent and Trade Mark Office (USPTO) and MAtrixware REsearch Collection (MAREC) patent document collection to retrieve the data. Other conventional data sources include clinical datasets (Dong et al., 2014), Gene Expression Omnibus (GEO) database (Hristovski et al., 2010), ArrayExpress (AE) database (Maver et al., 2013), Manually Annotated Target and Drug Online Resource (MATADOR) (Crichton et al., 2018), Biological General Repository for Interaction Datasets (BioGRID) (Crichton et al., 2018), PubTator (Crichton et al., 2018), Online Mendelian Inheritance in Man (OMIM) (Cohen et al., 2010) and TREC (Symonds et al., 2014).

Few non-English data sources such as Chinese Social Sciences Citation Index (Su and Zhou, 2009), China Biology Medicine disks (Qian et al., 2012), Chinese Medicine Library and Information System (Yao et al., 2008), Traditional Chinese Medicine Database (Gao et al., 2016) and Chinese Journal Full-text database (Yao et al., 2008) have also been utilised in LBD workflow.

The studies that have attempted to perform LBD in a non-traditional setting have extracted data from a variety of sources such as Twitter (Bhattacharya and Srinivasan, 2012), DailyMed: FDA drug labels (Bisgin et al., 2011), Google news (Maclean and Seltzer, 2011), and World Wide Web (WWW) (Gordon et al., 2002).

\section{PROCESS COMPONENT}

This section outlines the two major elements of the process component; filtering techniques and ranking/thresholding techniques. Moreover, this section also discusses about the resources utilised in LBD workflow.

\section{What are the filtering techniques used in the LBD process?}

It is vital to provide a concise output to the user that is easily interpretable by only including the most promising knowledge associations. To achieve this, the search space of the knowledge discovery should be reduced by eliminating spurious, general, uninteresting, or invalid terms/concepts. Different filtering techniques used in the literature are summarised in Figure 3 (a). 


\footnotetext{
${ }^{1}$ https:// semanticnetwork.nlm.nih.gov/SemanticNetworkArchive.html

${ }^{2}$ https://semanticnetwork.nlm.nih.gov/download/SemGroups.txt
} box-plot as the general terms removal mechanism. broader semantic category is picked, it may not filter out all the meaningless associations. subject and object of the predication (i.e. subject-relation-object triplet) (Hristovski et al., 2010). the starting term, thus, do not form any interesting association. (Liang et al., 2013), HUGO (Özgür et al., 2011) have been utilised.

Stop word Removal: Stop words typically denote non-topic general English terms. However, it could also include general terms used in the domain. For example, terms such as "drug", "treatment" can be considered as general terms in the medical domain. Using stop words to remove uninformative terms is a popular filtering technique used (Lever et al., 2017; Preiss and Stevenson, 2017; Sebastian et al., 2017b). Stop word list could be either manually created, obtained from other resources, or automatically generated. 1) Manually created: A popular example of this category is the stop word list created for the Arrowsmith project (Smalheiser, 2005) that have nearly 9500 terms by 2006 (Preiss and Stevenson, 2017). However, manual development of a stop words is costly, and time-consuming. Moreover, since these stop word lists are highly domain dependent, their applicability is also limited. 2) Obtained from other resources: Other resources used to obtain stopword list include NLTK toolkit (Lever et al., 2017) and Corpus of Contemporary American English (Lever et al., 2017), and Nvivo (Kibwami and Tutesigensi, 2014). 3) Automatically generated: Some studies (Preiss and Stevenson, 2017; Hu et al., 2010) have automatically created their stop word lists by employing different techniques. The most common way is eliminating terms that appear above a user-defined threshold (Pratt and Yetisgen-Yildiz, 2003). Different to the threshold-based removal, Xun et al. (2017) have followed Law of conformity to remove general terms by analysing the temporal change of terms, and Jha et al. (2016b) have considered outliers of the

Semantic Category Filter: This technique typically utilises the semantic type or group information provided by UMLS (Lever et al., 2017; Vlietstra et al., 2017). UMLS currently provides 127 semantic types $^{1}$ and each medical concept is classified to one or more of these semantic types based on the relevance. Each semantic type is further classified into one or more of 15 UMLS semantic groups ${ }^{2}$. For example, panic disorder belongs to the semantic type Mental or Behavioural Dysfunction and migraine belongs to the semantic type Disease and Syndrome. Both these semantic types belong to the semantic group of Disorders (Yetisgen-Yildiz and Pratt, 2009). This filtering technique involves imposing selected semantic type or group to restrict the linking and target concepts of the knowledge discovery process. However, selecting the most suitable semantic type or group is very challenging as it varies according to the problem. If a too granular semantic category is selected, it may also remove the valid associations, and if a too

Relation/predicate Type Filter: This filtering technique mostly consider the predications assigned using SemRep (Cameron et al., 2015; Rastegar-Mojarad et al., 2015). The typical procedure is to restrict the search space by eliminating uninteresting predicate types. For example, Cohen et al. (2010) have removed "PROCESS_OF" predication in their LBD process as it is less informative. Other types of predicate filtering techniques are; 1) removal of negated relations (Rastegar-Mojarad et al., 2016), 2) considering the directionality of the predicate (Baek et al., 2017), and 3) restricting the semantic type or group of the

Hierarchical Filter: This technique utilises the hierarchical information such as levels and relationships of terms to filter out uninformative associations (Shang et al., 2014). The levels of UMLS/MeSH hierarchy are typically examined to remove broader terms. For example, Qian et al. (2012) have eliminated terms in the first and second level of MeSH tree to remove less useful, broad associations. Another approach is to analyse the hierarchical relationships of the concepts to eliminate terms that are too close to the starting term. For instance, Pratt and Yetisgen-Yildiz (2006) have eliminated terms in the UMLS hierarchy such as children, siblings, parents and grandparents as they have observed that these terms are closely related to

Synonym Mapping: Mapping synonyms by grouping exactly or nearly equal terms of a given term is another technique used to reduce the results (Lever et al., 2017; Baek et al., 2017). To facilitate this, resources such as UMLS (Preiss et al., 2015), MeSH (Van der Eijk et al., 2004), Entrez gene database

POS Tag-based Filter: Several studies have utilised POS tags to restrict the search space by limiting the terms to nouns (Qi and Ohsawa, 2016), nominal phrases (Ittipanuvat et al., 2014) or verbs (Kim et al., 
2016). For example, Qi and Ohsawa (2016) have only extracted nouns as unigrams.

Template-based Restriction: Some studies (Maver et al., 2013; Cohen et al., 2012) have reduced the search space by only extracting the associations that adhere to the imposed rules/templates. For example, two forms of discovery patterns were defined by Hristovski et al. (2006) to restrict the detected associations that are in accordance with the templates of the two defined patterns.

Time-based Filter: Smalheiser (2005) have considered the time factor of the associations to reduce the search space of results. More specifically, given a user-defined year, only the associations that appear first time after the year (or before) have been considered as a filter. In addition, monitoring the temporal behaviours of words (Xun et al., 2017) have also been used to remove unnecessary terms.

Common Base Form: Deriving a common base form of the term to reduce the vocabulary space is another technique used in the literature. To facilitate this, the two popular techniques; stemming (Sebastian et al., 2015) and lemmatisation (Song et al., 2015) have been used in the literature.

Article Retrieval Filter: Several studies (Cherdioui and Boubekeur, 2013; Ittipanuvat et al., 2014) have attempted to limit the number of articles that need to be analysed through the LBD process to reduce the search space. For instance, Petric et al. (2012) have only considered the outlier documents for analysis without analysing all the documents derived from the search query.

Sentence Filter: Some studies (Hossain et al., 2012; Özgür et al., 2010) have only picked specific sentences from the text to analyse. For example, Ozgur et al. (2010) have only picked sentences from abstracts that describe gene interactions for the analysis. For a sentence to qualify as a potential interaction sentence, the authors have followed a rule-based mechanism. Moreover, Hossain et al. (2012) have employed machine learning to select sentences by training a Naïve Bayes classifier to differentiate context and results sentences in abstracts.

Network-based Filter: The network-based LBD approaches have utilised different techniques to reduce the size of the network. For example, Cairelli et al. (2015) have filtered their network by setting degree centrality and edge-occurrence frequency thresholds. Furthermore, Kastrin et al. (2014) have performed Pearson's Chi-Square test to detect if a particular connection occurs more often by chance. Ittipanuvat et al. (2014) have removed nodes that are not connected with any node in Largest Connected Components (LCC) of the graph.

Term Restrictions: Some studies have restricted terms in word-level and character-level to reduce the vocabulary space. Removal of unigrams from the analysis can be considered as an example for word-level restriction (Thaicharoen et al., 2009; Gordon et al., 2002). LBD studies (Roy et al., 2017; Kibwami and Tutesigensi, 2014) that have removed terms less than three characters in their LBD process can be considered as an example for character-level restrictions. However, since this filter does not consider semantic aspects of the terms into consideration, valuable short terms will be removed from the vocabulary.

Cohesion-based filter: Given two linking terms that are most similar, Smalheiser (2005) hypothesises that the term with a more narrow focus is the most useful. Hence, this filter calculates a cohesion score to select most granular-level terms as the results.

Expert/user-based filtering: Expert/user-based filtering (Gubiani et al., 2017; Preiss and Stevenson, 2017) involves the decision of an expert/user to remove uninteresting associations. For example, most of the semantic category filter requires user-defined semantic types/groups to perform the filtering. As described in 'Semantic Category Filter' technique, this selection is crucial as a more restrictive semantic category selection would risk at losing valid and informative associations whereas less restrictive semantic category selection would result in a noisy output. As a result, the success of these approaches greatly depends on the experience and prior knowledge of the user.

\section{What are the ranking/thresholding mechanisms used in LBD literature?}

Term ranking/thresholding is an important component of the LBD process as it should downweigh or remove noisy associations and upweight or retain the interesting and significant knowledge associations when ordering the terms. More specifically, these measures are used in two ways. 1) Thresholding: prune 


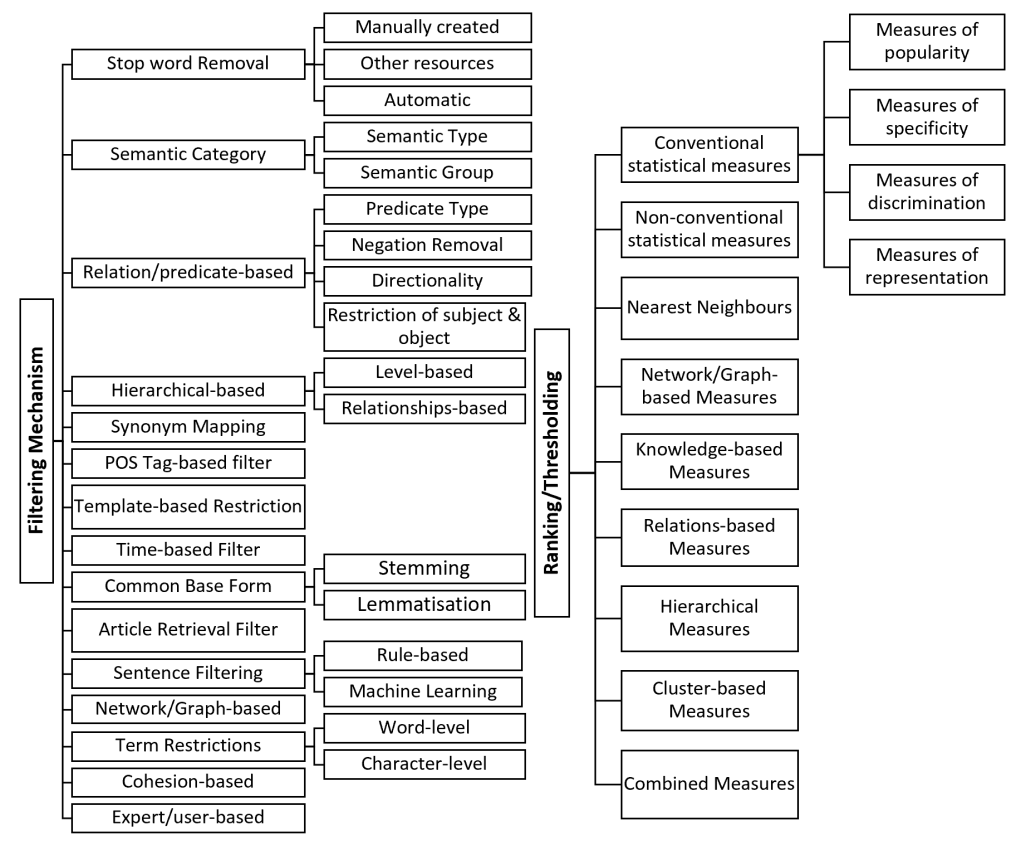

(a) (b)

Figure 3. (a) Filtering techniques, (b) Ranking/thresholding techniques

away uninteresting associations during the filtering process (e.g., setting a threshold to remove general terms), 2) Ranking: rank the selected set of associations based on their significance (e.g., rank the most frequent terms in the top of the list). Outlined below are the ranking schemes used in the discipline (See Figure 3 (b)).

Considering conventional statistical measures to rank/threshold terms is common in literature. These measures can be broadly divided into four categories (Aizawa, 2003) based on how they are mathematically defined; 1) Measures of popularity: these measures denote the frequencies of terms or probability of occurrences (e.g., concept frequency), 2) Measures of specificity: this category denotes the entropy or the amount of information of terms (e.g., mutual information), 3) Measures of discrimination: how terms are contributing to the performance of a given discrimination function is represented through these measures (e.g., information gain), and 4) Measures of representation: these measures denote the usefulness of terms in representing the document that they appear (e.g., TF-IDF).

Examples for conventional statistical measures used in LBD studies are; Token frequency (Gordon and Lindsay, 1996), Average token frequency (Ittipanuvat et al., 2014), Relative token frequency (Lindsay, 1999), Document/record frequency (Gordon and Lindsay, 1996), Average Document Frequency (Ittipanuvat et al., 2014), Relative Document Frequency (Thaicharoen et al., 2009), TF-IDF (Maciel et al., 2011), Mutual Information (Loglisci and Ceci, 2011), z-score (Yetisgen-Yildiz and Pratt, 2006), Information Flow (Bruza et al., 2006), Information Gain (Pusala et al., 2017), Odds Ratio (Bruza et al., 2006), Log Likelihood (Bruza et al., 2006), Support (Hristovski et al., 2005), Confidence (Hristovski et al., 2003), F-value of support and confidence (Hu et al., 2010), Chi-Square (Jha and Jin, 2016b), Kulczynski (Jha and Jin, 2016a), Cosine (Baek et al., 2017), Equivalence Index (Stegmann and Grohmann, 2003), Coherence (Pusala et al., 2017), Conviction (Pusala et al., 2017), Klosgen (Pusala et al., 2017), Least Contradiction (Pusala et al., 2017), Linear-Correlation (Pusala et al., 2017), Loevinger (Pusala et al., 2017), Odd Multiplier (Pusala et al., 2017), Piatetsky-Shapiro (Pusala et al., 2017), Sebag-Schoenauer (Pusala et al., 2017), Zhang (Pusala et al., 2017), Jaccard Index (Yang et al., 2017), Dice Coefficient (Yang et al., 2017), and Conditional Probability (Seki and Mostafa, 2009).

Additionally, non-conventional statistical measures such as such as Average Minimum Weight (AMW) (Yetisgen-Yildiz and Pratt, 2009), Linking Term Count with AMW (LTC-AMW) (Yetisgen-Yildiz and Pratt, 2009), Averaged Mutual Information Measure (AMIM) (Wren, 2004), Minimum Mutual Information Measure (MMIM) (Wren, 2004) have also been proposed in discipline to rank the potential associations. 
In comparison with AMW and Literature Cohesiveness, Yetisgen-Yildiz and Pratt (2009) have reported that they gained improved performance with $L T C-A M W$ measure (Swanson et al., 2006). Other types of ranking and thresholding categories used in LBD literature are summarised below.

Nearest Neighbours: In this category, the score of the association is decided by analysing its nearest neighbours. Such analysis is typically performed in distributional semantic models by employing measures such as Cosine (Gopalakrishnan et al., 2017), Euclidian distance (Van der Eijk et al., 2004), and information flow (Bruza et al., 2006).

Network/Graph-based Measures: Network/graph-based measures analyse node and edge-level attributes to score the associations. Examples of measures that represent this category include Degree centrality (Goodwin et al., 2012), Eigenvector centrality (Özgür et al., 2010), Closeness centrality (Özgür et al., 2011), Betweenness centrality (Özgür et al., 2010), Common Neighbours (Kastrin et al., 2014), Jaccard Index (Kastrin et al., 2014), Preferential Attachment (Kastrin et al., 2014), Personalised PageRank (Petric et al., 2014), Personalised Diffusion Ranking (Petric et al., 2014), and Spreading Activation (Goodwin et al., 2012).

Knowledge-based Measures: This category denotes the scoring measures such as MeSH-based Literature cohesiveness (Swanson et al., 2006), semantic type co-occurrence (Jha and Jin, 2016b), chemDB atomic count (Ijaz et al., 2010), and chemDB XLogP (Ijaz et al., 2010) that involve the knowledge from structured resources to rank the associations. The advantage of these measures is that they entangle semantic aspects into consideration to decide the potentiality of the association.

Relations-based Measures: Relations/predicate based measures (a sub-class of knowledge-based measures) analyse the relations extracted from resources such as SemRep to rank/threshold associations. Scoring measures such as Semantic relations frequency (Hristovski et al., 2010), Predicate independence (Rastegar-Mojarad et al., 2015), Predicate interdependence (Rastegar-Mojarad et al., 2015), Edge frequency-based weight (Kim et al., 2016), Edge traversal probability (Vlietstra et al., 2017), Relationship traversal probability (Vlietstra et al., 2017), Source traversal probability (Jha and Jin, 2016b), and Impact Factor (Huang et al., 2016) are examples of this category.

Hierarchical Measures: This category is another sub-class of knowledge-based measures that utilise hierarchical information of taxonomies such as $U M L S$, and $M e S H$ to derive the rankings. Child-to-parent and parent-to-child predications (Seki and Mostafa, 2009), and MeSH tree code depth (Gopalakrishnan et al., 2017) can be considered as examples.

Cluster-based Measures: In this category, cluster similarities are measured using techniques such as Intra-cluster similarity (Cameron et al., 2015), Jaccard Index (Ittipanuvat et al., 2014), Inclusion Index (Ittipanuvat et al., 2014), Dice coefficient (Ittipanuvat et al., 2014), Cosine (Ittipanuvat et al., 2014), Cosine similarity of tf-idf (Ittipanuvat et al., 2014), and Cosine similarity of tf-lidf (Ittipanuvat et al., 2014) to derive the ranking scores of associations.

Combined Measures: The idea of combined measures is to integrate multiple characteristics of an association to decide its potential ranking. For example, Torvik et al. (2007) have utilised machine learning techniques to combine seven characteristics of an association such as absolute and relative term frequencies, cohesion, recency, etc to obtain the final ranking score. Song et al. (2015) have also proposed a combined ranking measure by considering an average of three semantic similarity measures, and SemRep score. The characteristics that have been considered in the study of Ijaz et al. (2010) include UMLS semantic type, structural similarity, chemDB atomic count, and chemDB XLogP. Similarly, Gopalakrishnan et al. (2017) have also introduced a combined ranking measure by integrating global (node centrality and MeSH tree code depth) and local (semantic co-occurrence and betweenness centrality) measures. Overall, combined ranking measure are more flexible as they rely on multiple characteristics to prioritise the derived associations. 


\section{What are the domain independent and domain dependent resources utilised in LBD re- search?}

\section{Domain Dependent Resources}

Since the majority of LBD research are in Medicine, we refer medical resources as domain dependent resources. These resources are further categorised as; 1) Resources that provide background domain knowledge, and 2) Resources that are used in content analysis.

Resources to acquire background domain knowledge: The main purposes of extracting the domain knowledge are; 1) input data preparation (e.g., concept extraction), 2) filtering the noisy, uninteresting or unrelated associations (e.g., semantic type filtering), 3) prepare a ranking mechanism (e.g., hierarchical ranking), 4) evaluate the results (e.g., compare results with curated databases), and 5) training data preparation. The popular domain dependent resources used in the discipline are;

- UMLS: Lever et al. (2017); Vlietstra et al. (2017); Preiss and Stevenson (2017)

- MeSH: Baek et al. (2017); Xun et al. (2017); Pusala et al. (2017)

- SemMedDB/Semantic Medline: Vlietstra et al. (2017); Cairelli et al. (2015)

- Gene Ontology: Baek et al. (2017); Huang et al. (2016); Kim et al. (2016)

- Entrez Gene Database: Baek et al. (2017); Liang et al. (2013); Kwofie et al. (2011)

- Kyoto Encyclopedia of Genes and Genomes (KEGG): Kwofie et al. (2011)

- HGNC/HUGO: Petric et al. (2014); Ding et al. (2013); Maciel et al. (2011)

- UNIPROT: Baek et al. (2017); Vlietstra et al. (2017), Swiss-Prot Jelier et al. (2008)

- Therapeutic Target Database (TTD): Yang et al. (2017); Maciel et al. (2011)

- LocusLink: Smalheiser (2005); Hristovski et al. (2003)

- Online Mendelian Inheritance in Man (OMIM) Hristovski et al. (2003); Wren et al. (2004)

- Drug Bank: Vlietstra et al. (2017); Maciel et al. (2011); Ding et al. (2013)

- Comparative Toxicogenomics Database (CTD): Vlietstra et al. (2017); Yang et al. (2017)

- BioGRID: Huang et al. (2016); Crichton et al. (2018)

- Gene2pubmed: Cheung et al. (2012); Roy et al. (2017)

- Drugs.com: Maciel et al. (2011); Banerjee et al. (2014)

- SIDER Side Effect Resource: Vlietstra et al. (2017); Shang et al. (2014)

Additionally, other medical resources such as Medical Dictionary for Regulatory Activities (MedDRA) (Bisgin et al., 2011), Reactome Pathway Database (Kwofie et al., 2011), Orphanet (Baek et al., 2017), Human Metabolome Database (HMDB) (Baek et al., 2017), Lipid Maps (Baek et al., 2017), MassBank (Baek et al., 2017), DailyMed (Vlietstra et al., 2017), miRBase (Huang et al., 2016), miRGate (Huang et al., 2016), Transcriptional Regulatory Relationships Unraveled by Sentence-based Text mining (TRRUST) (Huang et al., 2016), PAZAR (Huang et al., 2016), Biomedical Knowledge Repository (BKR) (Cameron et al., 2015), MEDI (Shang et al., 2014), Tanabe-Wilbur list (Smalheiser, 2005), ChemDB (Ijaz et al., 2010), BioVerb (Kim et al., 2016), AIMED (Özgür et al., 2010), CB (Özgür et al., 2010), STRING (Petric et al., 2014), ToppGene (Petric et al., 2014), Endeavour (Petric et al., 2014), MIPS (Liang et al., 2013), Proteomics Standards Initiative Molecular Interactions (PSI-MI) (Song et al., 2015), Cell Line Knowledge Base (CLKB) (Song et al., 2015), Observational Medical Outcomes Partnership (OMOP) (Mower et al., 2016), METADOR (Crichton et al., 2018), Animal Transcription Factor Database (AnimalTFDB) (Roy et al., 2017), RxNorm (Malec et al., 2016), Vaccine Ontology (VO) (Özgür et al., 2011), Gene Reference Into Function (GeneRIF) (Cheung et al., 2012), Homologene (Jelier et al., 2008), Pharmacogenomics Knowledgebase (PharmGKB) (Kim and Park, 2016), Chinese Medical Terminology (Qian et al., 2012), Food and Drug Administration approved drug names (Wren, 2004), Rush University Medical Center's health encyclopedia (Banerjee et al., 2014) have also been employed in LBD workflow.

Our analysis reveals that $U M L S$ and $M e S H$ are most extensively used as the domain dependent resources in the literature ${ }^{3}$. The databases such as SemMedDB/Semantic Medline, Gene Ontology, Entrez Gene Database and $H U G O / H G N C$ are also popular among other resources. 
Resources for content analysis: The following resources have been used in LBD systems to process and analyse the content.

- MetaMap (Medical concept extraction): Preiss and Stevenson (2017, 2016); Cairelli et al. (2015)

- SemRep (Semantic predications extraction): Vlietstra et al. (2017); Preiss et al. (2015)

- Genia Tagger (Biological NER): Lever et al. (2017); Özgür et al. (2010)

- ABNER (Biological NER): Liang et al. (2013)

- Peregrine software (Biological NER): Jelier et al. (2008)

- DAVID tool (Gene annotation enrichment analysis): Maver et al. (2013); Özgür et al. (2010)

- RankProd Package (Meta analysis): Maver et al. (2013)

- BioTeKS Text Analysis Engine (Text annotation): Berardi et al. (2005)

- PubTator (PubMed citations annotation): Crichton et al. (2018)

- MedLEE (Structure and encode clinical reports): Malec et al. (2016)

- BioMedLEE (Semantic predications extraction): Hristovski et al. (2006)

- EpiphaNet (Interactive visual representation): Malec et al. (2016)

- SciMiner (literature mining and functional enrichment analysis): Hur et al. (2010)

- Biovista (Drug repurposing, Systems literature analysis environment): Persidis et al. (2004)

Among the content analysis tools, we observed that MetaMap and SemRep are the most popular selections ${ }^{3}$. MetaMap is a tool that recognises $U M L S$ concepts in the text whereas SemRep is used to extract semantic predications from the text. The predications in SemRep are formal representations of text content that comprises of subject-predicate-object triplets.

\section{Domain Independent Resources}

In this section, we summarise the resources that can be used in a cross domain LBD setting. For Named Entity Recognition (NER) resources such as GATE (Loglisci and Ceci, 2011), PKDE4J (Baek et al., 2017), Open Calais (Jha and Jin, 2016a), Sementax (Jha and Jin, 2016a) and Lingpipe (Hossain et al., 2012) have been employed in LBD literature.

Other text analytics resources include NLTK: to identify Noun Phrases (Sebastian et al., 2017b) and stop words (Lever et al., 2017), ReVerb: to extract relations (Preiss et al., 2015), Stanford parser: for dependency tree parsing (Sang et al., 2015) and extract relations (Preiss et al., 2015), Stanford CoreNLP: for sentence boundary detection, POS tagging and lemmatisation (Song et al., 2015), WordNet: for word sense disambiguation (Sebastian et al., 2017b), RacerPro: for logical and rule-based reasoning (Guo and Kraines, 2009), Link Grammar Parser: for sentence parsing (Ijaz et al., 2010), Vantage Point: for document clustering, auto-correction mapping and factor matrix analysis (Kostoff, 2011), Nvivo: to extract terms, stop words, coding and matrix coding queries (Kibwami and Tutesigensi, 2014), CLUTO: for document clustering (Kostoff et al., 2008b), Lucene: for information retrieval (Malec et al., 2016), and OntoGen: for topic ontology construction (Petriě et al., 2009).

To facilitate tasks such as network construction and visualisation, the following resources have been utilised in the literature; Neo4j (Vlietstra et al., 2017), JUNG (Kim et al., 2016), Gephi (Song et al., 2015), NetworkX (Wilkowski et al., 2011), and Large Graph Layout (LGL) (Ittipanuvat et al., 2014).

The importance of using the aforementioned resources in the LBD system is that they support the system's functionality not only to medical domain, but also to a wide variety of other domains. To date, such domain independent LBD methodologies have been rarely experimented.

\section{OUTPUT COMPONENT}

This section discusses the existing LBD output types, their drawbacks and the important characteristics that need to be fulfilled in terms of output visualisation to meet the objectives of the discipline. 


\section{What are the visualisation techniques used to display the results in LBD research?}

The most commonly used output of LBD systems is a ranked list of associations (Gubiani et al., 2017; Baek et al., 2017) ${ }^{3}$ where the top associations reflect the most probable knowledge links. However, providing merely a ranked list may not be the best way of visualising the results due to the following two reasons; 1) ranked associations are isolated in nature and do not provide an overall picture of all the suggested associations, and 2) ranked associations do not reflect how they are linked with the start and/or target concepts to better understand the association. As a result, the user needs to manually analyse the ranked associations individually to get an overview of the entire results and to interpret the linkage of the proposed associations with the start and/or target concepts. This points out the importance of exploring better visualisation techniques that can reduce the manual walkthroughs the user requires to perform. Discussed below are other visualisation techniques employed in the literature.

Group based on semantic type: In Manjal LBD system (Srinivasan, 2004), the outputted terms are organised by UMLS semantic type and ranked based on its estimated potential within the semantic type.

Rank based on templates: SemBT LBD system (Hristovski et al., 2010) ranks the identified novel associations using frequency of semantic relations (relation triplets) by specifying the subject and object of the relation. Ijaz et al. (2010) have ranked the detected associations based on an information model that includes substance, effects, processes, disease and body part.

Graph-based visualisations: Several studies have utilised graphs to visualise their LBD results. For instance, Kim et al. (2016) have used directed gene-gene network to clearly illustrate the discovery pathways suggested by their LBD methodology. A more advanced graph-based visualisation was proposed by Cameron et al. (2015) that outputs multiple context driven sub-graphs. Since the graph is divided into subgraphs by grouping the paths with similar context, the results can be easily interpreted by the user.

Ranking the discovery pathways: From LBD perspective, this technique can also be viewed as the output of AnC model. While graph-based visualisations display graphs as output, this technique only lists down the potential paths from the graph. Examples of this category include the study of Wilkowski et al. (2011) where the graph paths with high degree centrality are shown as the output, and the study of Kim et al. (2016) that considers the shortest paths in the graph as the output.

Story chain construction: Hossain et al. (2012) have attempted to build story chains by focusing on biological entities in PubMed abstracts. Their storytelling algorithm provides new insights to LBD visualisation and can be viewed as a next step of the Ranking the discovery pathways technique.

Word clouds: Malec et al. (2016) have used word clouds to present their results where the font size is proportionate to term frequencies.

Matrix-like visualisation: Qi and Ohsawa (2016) have proposed a matrix-like visualisation to detect mixed topics of their experiments. Moreover, they have also performed a user-based evaluation by providing their visualisation to the users to detect and interpret the mixed topics.

Using Existing Tools: Some studies have utilised existing tools such as Semantic Medline (Miller et al., 2012), OntoGen (Petrič et al., 2012), and EpiphaNet (Cohen et al., 2009), Biolab Experiment Assistant (BAE) (Persidis et al., 2004) for LBD visualisation.

Improving output visualisation is an essential component of the LBD workflow as it highly influences the user acceptance of the system. However, the existing literature has a little contribution towards output visualisation. This suggests the importance of involving Human Computer Interaction (HCI) techniques in the field. Some important characteristics that should be taken into consideration when developing a visualisation technique are; 1) concise output, 2) easily interpretable, 3) less complex, 4) visually attractive, and 5) assist users to gain new insights. Moreover, it is also vital to evaluate the efficiency of the visualisation technique by performing user-based evaluations (Santos, 2008). For instance, organising sessions for the participants to use the LBD tool (Cohen et al., 2010), observing how they interact with the tool and obtaining their feedback. Santos (2008) suggests two types of participants for such evaluations; target users and graphic designers. The author points out that the target users will assist to elicit new ideas whereas graphic designers will detect problems and provide suggestions with visual aspects. Furthermore, 
another interesting avenue is to involve target users with different level of expertise (i.e. expert vs novice) to evaluate how users with each level of expertise interact and benefit with the LBD process (Qi and Ohsawa, 2016).

\section{EVALUATION COMPONENT}

\section{What are the LBD evaluation types and their domain dependencies?}

Evaluating the effectiveness of the LBD results is challenging and remains to be an open issue. The main reason for this is that the LBD process detects novel knowledge that has not been publicly published anywhere and thus needs to be proven that they are useful. Moreover, there are no comprehensive gold standard datasets or consistent formal evaluation approaches in LBD (Ganiz et al., 2005). This review provides an in-depth classification of the existing evaluation techniques as summarised below.

\section{Evidences-based Evaluation:}

This category of evaluation asserts if a given association is accurate by using evidence from reliable sources such as existing discoveries, literature or curated databases.

Replicating existing medical discoveries: By far, this is the most commonly used evaluation technique. It measures the capability of the LBD methodology to reproduce the popular historical discoveries (see Table 2). The most popular selections of discovery replication are Swanson's initial two medical discoveries; Raynaud $\leftrightarrow$ Fish Oil and Migraine $\leftrightarrow$ Magnesium ${ }^{3}$. The normal procedure used for discovery replication is to only use the literature before the original paper of discovery as the input data of the LBD process and to verify if the mentioned associations detected in the original paper could be replicated. For example, if we consider Swanson's Raynaud $\leftrightarrow$ Fish Oil to replicate, the literature prior to 1986 (the published year of the paper) should only be considered.

Table 2. Replicated discoveries in the literature

\begin{tabular}{|c|c|}
\hline Replicated Discovery & Past Studies \\
\hline Migraine $\leftrightarrow$ Magnesium & $\begin{array}{l}\text { Xun et al. (2017); Preiss and Stevenson (2017); Sebastian } \\
\text { et al. (2017b); Qi and Ohsawa (2016); Song et al. (2015) }\end{array}$ \\
\hline Raynaud $\leftrightarrow$ Fish Oil & $\begin{array}{l}\text { Xun et al. (2017); Preiss and Stevenson (2017); Sebastian } \\
\text { et al. (2017b); Song et al. (2015); Preiss et al. (2015) }\end{array}$ \\
\hline Indomethacin $\leftrightarrow$ Alzheimer’s & $\begin{array}{l}\text { Xun et al. (2017); Preiss and Stevenson (2017); Preiss } \\
\text { et al. (2015); Cameron et al. (2015); Sang et al. (2015) }\end{array}$ \\
\hline $\begin{array}{l}\text { Schizophrenia } \leftrightarrow \text { Calcium-Independent } \\
\text { Phospholipase A2 }\end{array}$ & $\begin{array}{l}\text { Xun et al. (2017); Preiss and Stevenson (2017); Preiss } \\
\text { et al. (2015); Cameron et al. (2015); Srinivasan (2004) }\end{array}$ \\
\hline Alzheimer's $\leftrightarrow$ Estrogen & $\begin{array}{l}\text { Preiss and Stevenson (2017); Preiss et al. (2015); Cameron } \\
\text { et al. (2015); Preiss (2014) }\end{array}$ \\
\hline Magnesium deficiency $\leftrightarrow$ Neurologic & $\begin{array}{l}\text { Preiss and Stevenson (2017); Preiss et al. (2015); Preiss } \\
\text { (2014) }\end{array}$ \\
\hline Thalidomide $\leftrightarrow$ Chronic Hepatitis C & Kwofie et al. (2011); Jelier et al. (2008) \\
\hline Testosterone $\leftrightarrow$ Sleep & Cameron et al. (2015); Goodwin et al. (2012) \\
\hline Somatomedin $\mathrm{C} \leftrightarrow$ Arginine & Swanson and Smalheiser (1997); Preiss (2014) \\
\hline Chlorpromazine $\leftrightarrow$ Cardiac Hypertrophy & Cameron et al. (2015) \\
\hline Diethylhexyl (DEHP) $\leftrightarrow$ Sepsis & Cameron et al. (2015) \\
\hline Sleep $\leftrightarrow$ Depression & Goodwin et al. (2012) \\
\hline
\end{tabular}

However, discovery replication may not be the most effective way of evaluating the LBD methodology due to the following reasons. 1) These existing discoveries have not developed rigorously as a gold standard (Ganiz et al., 2005). For example, in Swanson's Raynaud $\leftrightarrow$ Fish Oil discovery, he merely suggests three novel intermediate connections. No evidence suggest that these connections identified 
through his trial and error approach can be seen as the only existing novel associations that connect the two domains, 2) Only focusing on one particular discovery might result in a system that performs well for that problem, but not for other problems even in the same domain (overfitting) (Yetisgen-Yildiz and Pratt, 2009). For example, Swanson and Smalheiser (1997) have replicated medical discoveries to evaluate Arrowsmith LBD system. The overfitting of their model is proved by the failure of it in recognising the links of Somatomedin-C $\leftrightarrow$ Arginine (Swanson, 1990). As a result, it is important to accompany other evaluation techniques along with discovery replication to measure the true efficiency of the proposed methodology.

Time-sliced evaluation: Time-sliced method evaluates the ability of LBD methodology to predict future co-occurrences based on a time-sliced dataset (Lever et al., 2017; Yang et al., 2017). Currently this is the most objective evaluation technique in the discipline that attempts to alleviate the following key issues (Yetisgen-Yildiz and Pratt, 2009).

1) Discovery replication is limited to the associations defined in that particular discovery and merely evaluates the ability of the methodology to recreate these specific associations. As a result, the remaining associations in the LBD output are not assessed. This makes it difficult to estimate the overall performance of the LBD system. Instead, time-sliced evaluation evaluates the complete list of associations outputted from the LBD system. 2) Most of LBD systems consider one or two existing medical discoveries to replicate. Hence, the true generalisability of their methodologies is not reflected. To overcome this issue, time-sliced evaluation is designed in a way it is repeatable for many starting concepts without only limiting to one or two existing medical discoveries. For example, Yetisgen-Yildiz and Pratt (2009) have considered 100 starting concepts for evaluation. 3) When replicating existing medical discoveries, the required intermediate and target terms are known in advance. As a result, the parameters of the system can be tuned in a way to obtain these terms which result in a system that performs well only for that discovery, but not in other cases. However, time-sliced evaluation is independent of prior knowledge as it does not require to know the output in advance which assists to perform an unbiased evaluation. 4) When replicating medical discoveries or in expert-based evaluation, it is difficult to compare the performance of different LBD systems. For example, if two systems claim that they could successfully replicate a particular discovery, it is hard to determine the most efficient system. Similarly, when incorporating expert decisions for evaluation, it is hard to quantify the results and compare against other LBD systems. As a result, time-sliced evaluation provides a platform to quantitatively compare the LBD outcomes with other systems.

This technique requires a cut-off-date to divide the dataset into two segments named as pre-cut-off (data before the specified cut-off date) and post-cut-off (data after the cut-off date). The pre-cut-off segment is treated as the training set, where the LBD system is employed to output the potential novel associations. Afterwards, the post-cut-off segment is utilised to develop the gold standard dataset to evaluate the produced associations. The gold standard dataset is created by identifying associations present in the post-cut-off set and absent in the pre-cut-off set. More specifically, it verifies whether the identified potential associations from the LBD process have taken place in the future. Therefore, the selection of the cut-off-date is crucial because it decides the time period that turns a hypothesis into a true discovery (Yetisgen-Yildiz and Pratt, 2009).

Manual literature search: Some studies have verified whether the produced associations are meaningful by manually searching the research articles that provide evidences of the existence of the specified association (Yang et al., 2017; Xun et al., 2017).

Intersection evaluation: This approach checks if the identified association has been co-occurred with the initial concept in any of the literature databases (e.g., Web of Science) or other sources (e.g., UseNet), and remove already known associations to filter out the novel associations (Gordon et al., 2002; Bhattacharya and Srinivasan, 2012). Afterwards, filtered novel associations are qualitatively evaluated.

Derive reference sets from literature: In this technique, the methodology is evaluated by using reference sets created using past literature. For example, in the study of Vlietstra et al. (2017), they have developed the reference set from the results of a systematic literature review to compare their results. In the work of Bernstam et al. (2016), they have utilised curated drug-ADE associations of Patrick Ryan et al. (2013) as the reference set to facilitate comparison. 
Compare results with curated databases: Cross referencing the LBD output with existing curated databases to verify the validity of the results is another technique. For example, some studies (Rastegar-Mojarad et al., 2015; Cheung et al., 2012) have used drug-disease interactions in Comparative Toxicogenomics Database (CTD) to validate their results. Similarly, other databases such as SIDER2 (Shang et al., 2014), GEO (Faro et al., 2011), GAD (Seki and Mostafa, 2009), StringDB (Nagarajan et al., 2015) have also been used for validation.

Compare results using other resources: In contrast to curated databases, this technique uses other reliable sources such as websites to validate the results. For instance, Vidal et al. (2014) have considered the information published in Mayo Clinic public website as the ground truth to evaluate the effectiveness of their ranking technique.

\section{Comparison with baselines:}

Different baseline models have been considered for comparison as discussed below.

Comparison with existing LBD tools: Several studies have considered the output of the popular LBD tools as the baseline to compare their results. The LBD tools that have been considered for results comparison are; BITOLA (Lever et al., 2017), ARROWSMITH (Loglisci and Ceci, 2011), Manjal (Vidal et al., 2014), ANNI (Lever et al., 2017), FACTA+ (Lever et al., 2017).

Comparison with previous LBD techniques: In this evaluation method, popular techniques that have already been tested by several LBD studies are considered as a baseline to facilitate comparison. This includes techniques like Association Rule Mining (e.g., Apriori (Hu et al., 2010)), distributional semantic techniques (e.g., LSI and RRI (Hu et al., 2010)), lexical statistics (e.g., TF-IDF, token frequencies (Kim et al., 2016)), and bibliographic coupling (Sebastian et al., 2015).

Comparison with previous $L B D$ work: Several studies have recreated previous LBD methodologies as a baseline to compare their results. Recreating work of Gordon et al. (1996) for comparison in (Gordon and Dumais, 1998) and recreating work of Hristovski et al. (2001) for comparison in (Huang et al., 2005) can be considered as examples. Some studies have only recreated subsections of the previous methodologies to evaluate the corresponding sub-section of their methodology. For instance, in (Rastegar-Mojarad et al., 2016), they have compared their ranking method with Linking Term count in (Yetisgen-Yildiz and Pratt, 2006). Others have straightaway compared the results with the results of the previous methodologies. For example, Qi and Ohsawa (2016) have compared their results of Migraine $\leftrightarrow$ Magnesium rediscovery with five other previous work in terms of precision, recall and F-measure.

Comparison with other state-of-the-art methods: Some studies have compared their work with state-of-theart methods in the relevant disciplines that are not necessarily tested in LBD before. For example, Crichton et al. (2018) have considered Adamic-Adar, Common Neighbours and Jaccard Index to compare their results as these algorithms are considered to be competitive and challenging baselines in link prediction discipline.

\section{Expert-oriented Evaluation:}

Expert-based evaluation: In expert-based evaluation, typically one (Gubiani et al., 2017) or two (Baek et al., 2017) domain experts inspect the LBD output to verify if the produced associations are meaningful. Alternatively, the domain expert may provide with a more open-ended evaluation (Gordon et al., 2002) by asking to provide anticipated future associations in the domain without actually looking at the LBD results. Afterwards, the list of potential associations provided by the expert is cross-checked against the actual LBD outcome. However, expert-based evaluation is expensive, time-consuming and suffers from subjectivity.

Qualitative analysis of selected results: A commonly used technique in LBD evaluation is qualitative analysis of the LBD output as an ad-hoc basis by the author(s) or domain expert(s) (Jha and Jin, 2016a; Huang et al., 2016). Since the complete LBD result is not properly evaluated, it is hard to determine the true accuracy of the LBD methodology. Moreover, same as in expert-based evaluation, the analysis of results suffers from subjectivity. 


\section{User-oriented Evaluation:}

It is crucial to perform user-oriented evaluations to verify the use of the LBD system for real-world usage. However, such evaluations are rarely performed in existing literature.

User-based evaluation: Evaluating user's ability to identify and formulating hypotheses from the output of the LBD process is an essential evaluation approach. However, such user-oriented evaluations are mostly neglected in LBD literature. As defined in the study (Qi and Ohsawa, 2016), criterions such as, utility (how useful is the generated hypothesis is?), interestingness (how interesting is the generated hypothesis?) and feasibility (to what extent the generated hypothesis can be realised?) can be utilised to score this user formulated hypotheses. These scores can be analysed to verify to what extent the LBD system assist the users to create scientifically sensible novel research hypotheses.

User-experience evaluation: Analysing how users interact with the LBD system plays a critical role as such user behaviours provide useful clues to improve the visualisation techniques of LBD results, user-interface, and the process of knowledge discovery. However, user-experience is rarely measured in LBD research. Qi and Ohsawa (Qi and Ohsawa, 2016) have compared the performance of experts and non-experts with their matrix-like visualisation LBD process and verified that the users with no prior knowledge also benefit from their LBD process. Similarly, a user performance evaluation was conducted in the study of Trevor et al. (2010) using one domain expert and one advanced undergraduate student using a total of nearly 6.5 hours of sessions to qualitatively evaluate their LBD tool named as EpiphaNet from the user's perspective.

\section{Proven from Experiments:}

Some studies have experimented the produced hypotheses to prove their validity. Since most of the LBD methodologies are in medical domain, clinical trials are mostly used to verify the derived hypotheses. However, validating all the derived associations of the LBD process using laboratory experiments is infeasible. Hence, the most likely to be successful association from the top of the list is picked to prove (Baek et al., 2017). As a result, this evaluation does not assess the accuracy of the remaining associations, thus, does not reflect the overall performance of the LBD methodology.

\section{Scalability Analysis:}

From query to query, the number of records that need to be analysed vary (Spangler et al., 2014). Therefore, it is important to measure how much time and storage required for the different phases of the LBD process to make the methodology more user-friendly.

Processing time analysis: Less processing time is a critical characteristic of the LBD process as the users would like to quickly obtain results for their queries. However, the time complexity is rarely measured and compared against other LBD methodologies in the literature. Few LBD studies (Hossain et al., 2012; Loglisci and Ceci, 2011) have performed processing time analysis of their algorithm.

Storage analysis: Analysing memory requirements is important when dealing with large datasets. In the study of Symond et al. (2014), they have analysed the storage complexity of several distributional models. Through their analysis, they have identified that Tensor Encoding model is well suited for open discovery as it is efficient in storing and computing independent of the vocabulary size.

\section{Evaluate Ranking Technique:}

The algorithm used to rank the detected associations plays a vital role in LBD methodology. It should rank the most promising associations in the top of the list by filtering the weak or false-positive associations. Therefore, the success of the LBD process greatly depends on the effectiveness of the ranking algorithm.

Evaluate ranking positions: Most of the studies have evaluated the ranking positions of the LBD output to verify the effectiveness of their ranking algorithm. For instance, the LBD studies that have chosen to replicate previous medical discoveries (Gordon and Dumais, 1998; Lindsay, 1999) have attempted to obtain the associations of that particular medical discovery in the top of the list. Some studies have compared their ranked list with a ranking list of previously published LBD studies to determine the superiority of their algorithm (Gordon and Dumais, 1998). Moreover, in techniques such as time-sliced evaluation (Yetisgen-Yildiz and Pratt, 2009), the efficiency of the ranking algorithm is measured by using information retrieval metrics such as 11-point average interpolated precision, precision at $\mathrm{k}$, and mean 
Table 3. Domain dependency of the evaluation techniques

\begin{tabular}{|c|c|c|}
\hline Evaluation Technique & Category1 & Category 2 \\
\hline \multicolumn{3}{|l|}{ Evidences-based Evaluation: } \\
\hline Replicating existing medical discoveries & $\checkmark$ & - \\
\hline Time-sliced evaluation & - & $\checkmark$ \\
\hline Manual literature search & - & $\checkmark$ \\
\hline Intersection evaluation & - & $\checkmark$ \\
\hline Derive reference sets from literature & - & $\checkmark$ \\
\hline Compare results with curated databases & $\checkmark$ & - \\
\hline \multicolumn{3}{|l|}{ Comparison with baselines: } \\
\hline Comparison with existing LBD tools & $\checkmark$ & - \\
\hline Comparison with previous LBD techniques & - & $\checkmark$ \\
\hline Comparison with previous LBD work & - & $\checkmark$ \\
\hline \multicolumn{3}{|l|}{ Expert-oriented Evaluation: } \\
\hline Expert-based evaluation & - & $\checkmark$ \\
\hline $\begin{array}{l}\text { Qualitative analysis of several selected results } \\
\text { User-oriented Evaluation: }\end{array}$ & - & $\checkmark$ \\
\hline User-based evaluation & - & $\checkmark$ \\
\hline User-experience evaluation & - & $\checkmark$ \\
\hline \multicolumn{3}{|l|}{ Proven from Experiments: } \\
\hline Clinical Tests (or relevant other experiments) & - & $\checkmark$ \\
\hline \multicolumn{3}{|l|}{ Scalability Analysis: } \\
\hline Processing time analysis & - & $\checkmark$ \\
\hline Storage analysis & - & $\checkmark$ \\
\hline \multicolumn{3}{|l|}{ Evaluate Ranking Technique: } \\
\hline Evaluate ranking positions & - & $\checkmark$ \\
\hline Evaluation ranking scores & - & $\checkmark$ \\
\hline \multicolumn{3}{|l|}{ Evaluate the quality of the output: } \\
\hline Evaluate the interestingness of results & - & $\checkmark$ \\
\hline Evaluation of quality and coherence of stories & - & $\checkmark$ \\
\hline
\end{tabular}

average precision. Some studies have automatically created ground-truths using evidence from literature to evaluate their ranking algorithms (Xun et al., 2017).

Evaluate ranking scores: Mapping the ranking scores of the detected associations with scores obtained from databases (Baek et al., 2017) or other algorithms (Pusala et al., 2017) is another evaluation technique used in the literature.

\section{Evaluate the quality of the output:}

Evaluate the interestingness of results: Cameron et al. (2015) have used association rarity to statistically evaluate the interestingness of the LBD output. To facilitate this, they have queried Medline to obtain the number of articles that contain the derived associations and divided it by the number of associations. Afterwards, an interesting score was obtained which is proportionate to the rarity score.

Evaluation of quality and coherence of stories: This evaluation metric provides a novel perspective to LBD evaluation. The quality of the produced story chains can be evaluated using dispersion coefficient 
which is 1 for an ideal story (Hossain et al., 2012). This type of evaluation can be adapted when the LBD methodology outputs a chain of story path (e.g., output of an AnC model).

We also analysed the generalisability of each evaluation technique across domains. To achieve this, the previously discussed evaluation techniques are categorised into the following two groups; Category1: Highly domain dependent and only applicable to domains where similar resources are available, and Category2: Domain-independent (Table 3).

The most prominent and widely used evaluation technique which is discovery replication is only limited to the medical domain. Other popular evaluation techniques such as the use of curated databases and resources and comparison with existing LBD tools are also highly domain dependent and mostly available for the medical domain. Nevertheless, the most objective evaluation technique considered so far in the discipline, which is Time-sliced Evaluation is domain independent. Most of the remaining evaluation techniques are typically independent of the domain and can be utilised in non-medical LBD studies.

\section{What are the main quantitative measurements used to assess the effectiveness of the results?}

Different information retrieval metrics have been used to obtain a quantitative understanding of the performance of the LBD methodologies as summarised in Table 4. From our analysis ${ }^{3}$ we observed that precision (i.e. fraction of associations obtained from the LBD process that are relevant), recall (i.e. fraction of relevant associations that are successfully retrieved), F-measure (i.e. harmonic mean of precision and recall) and Area Under Curve $(A U C)$ (i.e. area under the Receiver Operating Characteristic (ROC) curve which falls in the range from 1 to 0.5 ) are the popular metrics used in the literature.

Table 4. Quantitative measures used in the literature

\begin{tabular}{|l|l|}
\hline Measure & Past Studies \\
\hline Precision & Lever et al. (2017); Yang et al. (2017); Preiss and Stevenson (2017) \\
Recall & Sebastian et al. (2017b); Jha and Jin (2016a); Sang et al. (2015) \\
F-Measure & Preiss et al. (2015); Sebastian et al. (2015); Sang et al. (2015) \\
Precision at k & Vlietstra et al. (2017); Shang et al. (2014); Song et al. (2015) \\
Recall at k & Lever et al. (2017); Vlietstra et al. (2017); Shang et al. (2014) \\
Average Precision & Cohen et al. (2012); Roy et al. (2017) \\
Mean Average Precision & Yang et al. (2017); Shang et al. (2014); Crichton et al. (2018) \\
Precision over time & Yetisgen-Yildiz and Pratt (2006) \\
Recall over time & Vlietstra et al. (2017); Yetisgen-Yildiz and Pratt (2006) \\
11-point average interpo- & Yetisgen-Yildiz and Pratt (2009) \\
lated precision & \\
Area Under Curve & Lever et al. (2017); Kastrin et al. (2016); Sebastian et al. (2015) \\
Accuracy & Sebastian et al. (2017b); Sang et al. (2015) \\
Cumulative Gain & Vlietstra et al. (2017) \\
Mean Reciprocal Rank & Song et al. (2015) \\
Correlation Analysis & Baek et al. (2017); Yang et al. (2017); Xun et al. (2017) \\
\hline
\end{tabular}

Since most of the time the users will not able to go through the entire list of suggested associations, it is also important to evaluate the proportion of associations in the top k positions that are relevant. For this purpose, the metrics such as precision at $k$, recall at $k, 11$-point average interpolated precision, and Mean Reciprocal Rank have been used in the literature. 


\section{LIMITATIONS}

Even though we present the insights gleaned from our rigorous literature analysis with confidence, we may have missed LBD research articles that are outside of the six databases and six keywords we used. To alleviate this issue to some extent, we also included the references from a recent review (Henry and McInnes, 2017) during our paper retrieval process as discussed in the Methods section.

\section{DISCUSSIONS AND FUTURE WORK}

The key findings and future research directions of each component of the LBD workflow are summarised below.

Input Component: The primary source of data utilised in LBD studies is research papers. Different studies have extracted different details from the research papers for analysis. Among them, using title and abstract is the most popular method. However, some studies have proven the use of full-text, and other metadata such as keywords, references, author details and venue details assist to glean additional clues of the anticipated knowledge links. Lee et al. (2015) point out that different perspectives are reflected by different data types used in the content of the research papers. In their analysis, they have found that keyphrases, citation relationships, and $\mathrm{MeSH}$ reflect the views of authors, citers, and indexers respectively. Moreover, Kostoff et al. (2004) have analysed the information content in various fields of a paper using four metrics; total number of phrases, number of unique phrases, factor matrix filtering and multi-link hierarchical clustering. They have identified that the selection of the field depends on the objectives of the study as described in (Kostoff et al., 2004). Hence, selecting the suitable data type in the papers in crucial as they represent different perspectives (Lee et al., 2015) and information content (Kostoff et al., 2004) and mainly depends on the objective of the research. Furthermore, Nagarajan et al. (2015) have discovered that the LBD performance mainly depends on the richness of the information being used.

Apart from research papers, several approaches have experimented the LBD process with other traditional data types such as patents and clinical case reports. Smalheiser et al. (2015) have identified that information nuggets (i.e. main findings) are surprisingly prevalent and large in clinical case reports. Mostly, the title itself reveals the main findings of the case report that enable ample opportunity for finding-based information retrieval (Smalheiser et al., 2015).

Interestingly, LBD methodology was successfully adopted to non-traditional data types such as drug labels, tweets, news articles, and web content. Therefore, an interesting future direction would be to analyse how the LBD process using research papers can be enhanced by integrating knowledge from non-traditional data types such as tweets. Furthermore, since most of the non-traditional data types are utilised in medical domain another interesting avenue would be integrate LBD process in other domains using data types such as product descriptions (for product recommendation), movie scripts (for movie recommendation), and recipe books (for recipe recommendation).

With respect to unit of analysis, making use of controlled vocabularies such as UMLS, MeSH, Entrez Gene to extract concepts is the most popular approach. However, research outside of medical domain have followed a term-based approach by extracting n-grams. As the controlled vocabularies utilised yet in LBD research are in the medical domain, an interesting future avenue is to experiment the use of general-purpose controlled vocabularies (such as DBpedia, Freebase, and YAGO) to facilitate knowledge discovery in a cross-disciplinary manner.

Process Component: Swanson's manually detected medical discoveries have set the base for the LBD research. Later various computational techniques such as statistical, knowledge-based, relations-based, hierarchical, graph-based, bibliometrics-based, link prediction etc. were proposed to automate the process of LBD. The filtering and ranking techniques used in the LBD methodology are two equally important major components of the LBD workflow.

Many of the filtering mechanisms utilised in LBD studies have restricted the search space using wordlevel filters. Considering the article-level filters (e.g., analysing the contribution of outlier documents), section-level filters (e.g., analysing the contribution of different sections in a research article such as introduction and conclusion), or sentence-level filters (e.g., analysing the contribution of sentences that describes the main findings) have received little attention in the literature. Therefore, analysing the effect of various article, section and sentence level filtering techniques to remove noisy associations before the word-level filtering is another important area that needs to be further explored. Ultimately, such 
techniques will also help to further narrow down the literature search and to eliminate the hindrances of the existing word-level filters.

As for the ranking techniques, most of the studies have utilised conventional statistical measures to rank/threshold their results. Whether using such single measure alone would be sufficient to rank the most promising associations in the top of the list is doubtful. In other words, an association may require satisfying several characteristics to become a significant and promising association among others. Therefore, it would be more interesting to develop a ranking approach that reflects the identified characteristics of potential associations to prioritise the results. For instance, Torvik et al. (2007) have attempted to derive a formula using seven features that capture various characteristics of an association into a single score by employing a machine learning model. Identifying the important characteristics of a significant and promising association and deriving a score based on these characteristics to rank the LBD results would be more successful than merely relying on standard single measures. In this regard, the analysis of different types of gaps in the literature is useful (Peng et al., 2017). Moreover, Smalheiser (2017) suggests the need of several ranking measures to customise the LBD output according to the user preferences. LION LBD system (Pyysalo et al., 2018) that supports multiple scoring functions to facilitate flexible ranking mechanism can be taken as an example.

Output Component: The typical output of the LBD process is a ranked list of terms that denote the potential associations. However, it is not an effective output technique as the users need to interpret the logical connections of the associations by manually reading the research articles which is difficult and time-consuming. As a result, other visualisation techniques such as term groupings, graphs, and discovery pathways have been proposed in LBD literature. However, the extent to which these proposed techniques assist the user has been rarely measured. Therefore, providing a better visualisation (which is concise, easily interpretable, less complex, visually attractive, and assist users to gain new knowledge) and measuring the user experience of the visualisation are two critical components of LBD workflow that need to be further explored by integrating $\mathrm{HCI}$ techniques.

Nevertheless, the importance of such techniques has been overlooked by the LBD community. To date, only a few LBD research (Wilkowski et al., 2011; Hristovski et al., 2006) have contributed in terms of user interaction studies. These studies make use of Information Foraging Theory which is a technique that analyses the user's information retrieval behaviour. The theory evaluates the user's information seeking behaviour in terms of costs and benefits. If the user can maximise his/her rate of gaining valuable information (i.e. maximum benefit) by spending the lowest amount of energy (i.e. minimum effort), it is called as an optimal foraging. The key concepts in an information-seeking context are information, information patches, information scents and information diet which needed to be supported effectively when designing interfaces (Ruthven and Kelly, 2011). Therefore, the challenge of information visualisation is to discover effective mechanisms to represent massive amounts of data and provide effective ways to navigate through them to support users with optimal foraging. The novel advances in HCI research will be useful in this regard (Stephanidis, 2019). Moreover, Smalheiser and Torvik (2008) emphasises the importance of simplicity in user-interfaces of LBD tools to support widening the target audience.

Evaluation Component: Evaluating the LBD output is challenging and remains to be an open issue as the field lacks gold standard datasets or consistent formal evaluation techniques. The most widely used evaluation technique is replicating Swanson's medical discoveries. However, relying merely on discovery replication can be restrictive and may fail to reflect the true performance of the LBD methodology. Hence, this technique should be accompanied with other evaluation techniques to overcome these limitations. Another popular technique is qualitatively evaluating the results randomly by an expert or author. Nevertheless, this does not give an overall image of the LBD system's performance as few valid associations are taken into consideration for discussion. An LBD system that produces a handful of valid associations in a sea of invalid associations tend to be inefficient (Yetisgen-Yildiz and Pratt, 2009). As a result, besides this random quantitative evaluation, the system should also be validated qualitatively to measure the overall performance of the system.

To date, time sliced evaluation is considered as the most objective evaluation technique proposed in the field. However, this evaluation technique suffers from two major limitations; 1) The association is proven to valid if the starting and linking term co-occur in future publications (that do not co-occur in the training set). However, co-occurrence does not necessarily mean that the proposed link has been 
established, and 2) Rejected associations can still be valid even though they have not been published yet.

To overcome the first limitation, it is important to perform much deeper analysis of language (Korhonen et al., 2014) to verify whether the co-occurrence imply a true association which can be considered as an interesting future direction. Additionally, some studies have attempted to utilise evidences from curated databases (e.g., CTD, StringDB) as an alternative for co-occurrence in time-sliced evaluation. However, such curated databases are limited to certain problems and may not be available for every domain or problem. The second limitation of time-sliced evaluation can be alleviated to some extent through domain expert involvement by further evaluating the rejected associations.

Another interesting direction for future evaluation is to incorporate the actual end users of LBD research to validate the results which is a neglected area in the literature. For instance, involving users with a diverse range of knowledge and expertise (e.g., novice to expert) will help to understand the extent to which each user will be benefited from the LBD output. In this regard, the hypotheses scoring mechanism used by Qi and Ohsawa (2016) can be considered as a successful first step.

Due to the massive influx of scientific knowledge, the volume of data that the LBD system expects to analyse increases with time. For instance, a simple search of "dementia" results in more than 150,000 records in PubMed alone. This highlights the importance of performing scalability analysis of the LBD systems in terms of time and storage. This will also improve the usability of the system.

\section{CONCLUSION}

In this review, we present novel, up-to-date and comprehensive categorisations to answer each of our research questions to provide a detailed overview of the discipline. The review summary and a comparison with the following recent reviews (Henry and McInnes, 2017; Gopalakrishnan et al., 2019) are available at https://tinyurl.com/workflow-summary.

With respect to input component, it is evident that LBD community is showing a growing research interest towards integrating knowledge from non-traditional data sources to enhance the traditional setting of the LBD framework and to explore new application areas. Nevertheless, the selection of the input needs to be precise and cross-checked against the research objectives as different data types reflect different perspectives (Lee et al., 2015) and information content (Kostoff et al., 2004). Filtering and ranking are two important constituents of the process component. Most of the filtering techniques examined in the discipline are at word-level. However, the importance of article-level, section-level and sentence-level filters have been rarely studied in the literature. Considering the ranking component, most of the studies have employed a single conventional ranking technique to prioritise the generated discoveries. This showcase the need of developing a series of interestingness measures that customise the LBD output that suit multiple scientific investigations (Smalheiser, 2012b).

The output component of LBD workflow, is largely neglected in the prevailing literature which emphasises the necessity of conducting user-interaction studies to assess the user experience. Concerning the evaluation component, time-sliced evaluation is the current most objective technique used to validate the results. However, this technique suffers from several limitations which suggests the requirement of developing new evaluation methods and metrics to evaluate the generated output.

We hope that the future LBD studies will contribute to overcome the prevailing research deficiencies in the LBD workflow with the ultimate intention of uplifting the typical research procedures which are followed by the scientists.

\section{REFERENCES}

Ahmed, A. (2016). Literature-based discovery: Critical analysis and future directions. International Journal of Computer Science and Network Security (IJCSNS), 16(7):11.

Aizawa, A. (2003). An information-theoretic perspective of tf-idf measures. Information Processing \& Management, 39(1):45-65.

Baek, S. H., Lee, D., Kim, M., Lee, J. H., and Song, M. (2017). Enriching plausible new hypothesis generation in PubMed. PLoS ONE.

Banerjee, R., Choi, Y., Piyush, G., Naik, A., and Ramakrishnan, I. (2014). Automated suggestion of tests for identifying likelihood of adverse drug events. In Healthcare Informatics.

Berardi, M., Lapi, M., Leo, P., and Loglisci, C. (2005). Mining generalized association rules on biomedical 
literature. In Industrial, Engineering and Other Applications of Applied Intelligent Systems, pages 500-509. Springer.

Bhattacharya, S. and Srinivasan, P. (2012). A semantic approach to involve twitter in lbd efforts. In $B I B M W$ 2012, pages 248-253. IEEE.

Bisgin, H., Liu, Z., Fang, H., Xu, X., and Tong, W. (2011). Mining fda drug labels using an unsupervised learning technique-topic modeling. In BMC bioinformatics, volume 12, page S11. BioMed Central.

Bruza, P., Cole, R., Song, D., and Bari, Z. (2006). Towards operational abduction from a cognitive perspective. Logic Journal of IGPL, 14(2):161-177.

Cairelli, M., Fiszman, M., Zhang, H., and Rindflesch, T. (2015). Networks of neuroinjury semantic predications to identify biomarkers for mild traumatic brain injury. Journal of biomedical semantics, $6(1): 25$.

Cameron, D., Kavuluru, R., Rindflesch, T. C., Sheth, A. P., Thirunarayan, K., and Bodenreider, O. (2015). Context-driven automatic subgraph creation for literature-based discovery. Journal of biomedical informatics, 54:141-157.

Cheadle, C., Cao, H., Kalinin, A., and Hodgkinson, J. (2017). Advanced literature analysis in a big data world. Annals of the New York Academy of Sciences, 1387(1):25-33.

Cherdioui, S. and Boubekeur, F. (2013). Information retrieval techniques for knowledge discovery in biomedical literature. In Programming and Systems (ISPS), 2013, pages 137-142. IEEE.

Cheung, W., Ouellette, B. F., and Wasserman, W. (2012). Inferring novel gene-disease associations using medical subject heading over-representation profiles. Genome medicine, 4(9):75.

Cohen, T., Schvaneveldt, R., and Rindflesch, T. (2009). Predication-based semantic indexing: permutations as a means to encode predications in semantic space. AMIA Апnи Sутр proc.

Cohen, T., Whitfield, G., Schvaneveldt, R., Mukund, K., and Rindflesch, T. (2010). Epiphanet: an interactive tool to support biomedical discoveries. Biomedical discovery and collaboration, 5:21.

Cohen, T., Widdows, D., Schvaneveldt, R. W., Davies, P., and Rindflesch, T. C. (2012). Discovering discovery patterns with predication-based semantic indexing. Journal of biomedical informatics, 45(6):1049-1065.

Cohen, T., Widdows, D., Stephan, C., Zinner, R., Kim, J., Rindflesch, T., and Davies, P. (2014). Predicting high-throughput screening results with scalable literature-based discovery methods. CPT: pharmacometrics \& systems pharmacology, 3(10):1-9.

Crichton, G., Guo, Y., Pyysalo, S., and Korhonen, A. (2018). Neural networks for link prediction in realistic biomedical graphs: a multi-dimensional evaluation of graph embedding-based approaches. BMC bioinformatics, 19(1):176.

Davies, R. (1989). The creation of new knowledge by information retrieval and classification. Journal of documentation, 45(4):273-301.

Ding, Y., Song, M., Han, J., Yu, Q., Yan, E., Lin, L., and Chambers, T. (2013). Entitymetrics: Measuring the impact of entities. PloS one, 8(8):e71416.

Dong, W., Liu, Y., Zhu, W., Mou, Q., Wang, J., and Hu, Y. (2014). Simulation of Swanson's literaturebased discovery: Anandamide treatment inhibits growth of gastric cancer cells in vitro and in silico. PLOS ONE.

Faro, A., Giordano, D., and Spampinato, C. (2011). Combining literature text mining with microarray data. Briefings in bioinformatics, 13(1):61-82.

Ganiz, M. C., Pottenger, W. M., and Janneck, C. D. (2005). Recent advances in literature based discovery. Journal of the American Society for Information Science and Technology, JASIST (Submitted).

Gao, H., Wang, Y., Tao, J., Liu, Z., Li, J., Yu, T., Yu, Q., Tian, Y., and Zhang, H. (2016). Cordycepssinensis may have a dual effect on diabetic retinopathy. In Proceedings ITME 2015.

Goodwin, J. C., Cohen, T., and Rindflesch, T. (2012). Discovery by scent: Discovery browsing system based on the information foraging theory. In BIBMW 2012, pages 232-239. IEEE.

Gopalakrishnan, V., Jha, K., Jin, W., and Zhang, A. (2019). A survey on literature based discovery approaches in biomedical domain. Journal of biomedical informatics, pages 103-141.

Gopalakrishnan, V., Jha, K., Xun, G., Ngo, H. Q., and Zhang, A. (2017). Towards self-learning based hypotheses generation in biomedical text domain. Bioinformatics, 34(12):2103-2115.

Gordon, M. and Dumais, S. (1998). Using latent semantic indexing for literature based discovery. Journal of the American Society for Information Science, 49(8):674-685.

Gordon, M., Lindsay, R. K., and Fan, W. (2002). Literature-based discovery on the world wide web. ACM 
Transactions on Internet Technology (TOIT), 2(4):261-275.

Gordon, M. D. and Lindsay, R. K. (1996). Toward discovery support systems: A replication, reexamination, and extension of Swanson's work on literature-based discovery of a connection between Raynaud's and fish oil. Journal of the American Society for Information Science, 47(2):116-128.

Gubiani, D., Fabbretti, E., Cestnik, B., Lavrač, N., and Urbančič, T. (2017). Outlier based literature exploration for cross-domain linking of Alzheimer's disease and gut microbiota. Expert Systems with Applications.

Guo, W. and Kraines, S. B. (2009). Discovering relationship associations in life sciences using ontology and inference. In KDIR, pages 10-17.

Henry, S. and McInnes, B. T. (2017). Literature based discovery: models, methods, and trends. Journal of biomedical informatics, 74:20-32.

Hossain, M. S., Gresock, J., Edmonds, Y., Helm, R., Potts, M., and Ramakrishnan, N. (2012). Connecting the dots between pubmed abstracts. PloS one, 7(1):e29509.

Hristovski, D., Friedman, C., Rindflesch, T., and Peterlin, B. (2006). Exploiting semantic relations for literature-based discovery. AMIA Annual Symposium Proceedings.

Hristovski, D., Kastrin, A., Peterlin, B., and Rindflesch, T. C. (2010). Combining semantic relations and dna microarray data for novel hypotheses generation. In Linking literature, information, and knowledge for biology, pages 53-61. Springer.

Hristovski, D., Peterlin, B., Mitchell, J. A., and Humphrey, S. M. (2005). Using literature-based discovery to identify disease candidate genes. Medical informatics, 74(2-4):289-298.

Hristovski, D., Peterlin, B., Mitchell, J. A., Humphrey, S. M., Sitbon, L., and Turner, I. (2003). Improving literature based discovery support by genetic knowledge integration. Stud Health Technol Inform, 95.

Hristovski, D., Stare, J., Peterlin, B., and Dzeroski, S. (2001). Supporting discovery in medicine by association rule mining in medline and umls. Studies in health technology and informatics, (2):13441348.

Hu, X., Zhang, X., Yoo, I., Wang, X., and Feng, J. (2010). Mining hidden connections among biomedical concepts from disjoint biomedical literature sets through semantic-based association rule. Intelligent Systems, 25(2):207-223.

Huang, W., Nakamori, Y., Wang, S. Y., and Ma, T. J. (2005). Mining medline for new possible relations of concepts. In Computational and Information Science, volume 3314, pages 794-799.

Huang, Y., Wang, L., sen Zan, and Lin (2016). ARN: Analysis and prediction by adipogenic professional database. BMC Systems Biology, 10(1).

Hur, J., Sullivan, K., Schuyler, A., Hong, Y., Pande, M., States, D., Jagadish, H., and Feldman, E. (2010). Literature-based discovery of diabetes-and ros-related targets. BMC medical genomics, 3(1):49.

Ijaz, A. Z., Song, M., and Lee, D. (2010). Mkem: a multi-level knowledge emergence model for mining undiscovered public knowledge. In BMC bioinformatics, volume 11, page S3. BioMed Central.

Ittipanuvat, V., Fujita, K., Sakata, I., and Kajikawa, Y. (2014). Finding linkage between technology and social issue: A literature based discovery approach. Journal of Engineering and Technology Management, 32:160-184.

Jelier, R., Schuemie, M. J., Veldhoven, A., Dorssers, L. C., Jenster, G., and Kors, J. A. (2008). Anni 2.0: a multipurpose text-mining tool for the life sciences. Genome biology, 9(6):R96.

Jha, K. and Jin, W. (2016a). Mining hidden knowledge from the counterterrorism dataset using graphbased approach. In Applications of Natural Language to Information Systems, pages 310-317. Springer.

Jha, K. and Jin, W. (2016b). Mining novel knowledge from biomedical literature using statistical measures and domain knowledge. In Bioinformatics, Computational Biology, and Health Informatics, pages 317-326. ACM.

Kastrin, A., Rindflesch, T., and Hristovski, D. (2016). Link prediction on a network of co-occurring mesh terms. Methods of information in medicine, 55(04):340-346.

Kastrin, A., Rindflesch, T. C., and Hristovski, D. (2014). Link prediction on the semantic medline network. In Discovery Science, pages 135-143. Springer.

Kibwami, N. and Tutesigensi, A. (2014). Using the literature based discovery research method in a context of built environment research. In Proceedings 30th Annual ARCOM Conference, volume 1, pages 227-236. ARCOM.

Kim, H. and Park, S. (2016). Discovering disease-associated drugs using web crawl data. In ACM Symposium on Applied Computing, pages 9-14. ACM. 
Kim, Y. H., Beak, S. H., Charidimou, A., and Song, M. (2016). Discovering new genes in the pathways of common sporadic neurodegenerative diseases: a bioinformatics approach. Journal of Alzheimer's Disease, 51(1):293-312.

Kim, Y. H. and Song, M. (2019). A context-based abc model for literature-based discovery. PloS one, 14(4):e0215313.

Korhonen, A., Guo, Y., Baker, S., Yetisgen-Yildiz, M., Stenius, U., Narita, M., and Liò, P. (2014). Improving literature-based discovery with advanced text mining. In International Meeting on Computational Intelligence Methods for Bioinformatics and Biostatistics, pages 89-98. Springer.

Kostoff, R., Block, J., Stump, J., and Pfeil, K. (2004). Information content in medline record fields. International Journal of Medical Informatics, 73(6):515-527.

Kostoff, R. N. (2011). Literature-related discovery: Potential treatments and preventatives for sars. Technological Forecasting and Social Change, 78(7):1164-1173.

Kostoff, R. N., Block, J. A., Stump, J. A., and Johnson, D. (2008a). Literature-related discovery (lrd): potential treatments for raynaud's phenomenon. Technological Forecasting and Social Change, 75(2):203-214.

Kostoff, R. N., Briggs, M. B., Solka, J. L., and Rushenberg, R. L. (2008b). Literature-related discovery (lrd): Methodology. Technological Forecasting and Social Change, 75(2):186-202.

Kwofie, S. K., Radovanovic, A., Sundararajan, V. S., Maqungo, M., Christoffels, A., and Bajic, V. B. (2011). Dragon exploratory system on hepatitis c virus (deshcv). Infection, Genetics and Evolution, 11(4):734-739.

Lee, D., Kim, W., Charidimou, A., and Song, M. (2015). A bird's-eye view of alzheimer's disease research. Journal of Alzheimer's Disease, 45(4):1207-1222.

Lever, J., Gakkhar, S., Gottlieb, M., Rashnavadi, T., Lin, S., Siu, C., Smith, M., Jones, M. R., Krzywinski, M., and Jones, S. J. (2017). A collaborative filtering-based approach to biomedical knowledge discovery. Bioinformatics, 34(4):652-659.

Liang, R., Wang, L., and Wang, G. (2013). New insight into genes in association with asthma: Literaturebased mining and network centrality analysis. Chinese Medical Journal.

Lindsay, R. K. (1999). Literature-based discovery by lexical statistics. Journal of the American Society for Information Science, 50(7):574-587.

Loglisci, C. and Ceci, M. (2011). Discovering temporal bisociations for linking concepts over time. In Joint European Conference on Machine Learning and Knowledge Discovery in Databases, pages 358-373. Springer.

Maciel, W., Faria-Campos, A., Gonçalves, M., and Campos, S. (2011). Can the vector space model be used to identify biological entity activities? In BMC genomics, volume 12. BioMed Central.

Maclean, D. and Seltzer, M. (2011). Mining the Web for Medical Hypotheses A Proof-of-Concept System. In HEALTHINF 2011, pages 303-308.

Malec, S. A., Wei, P., Xu, H., Bernstam, E. V., Myneni, S., and Cohen, T. (2016). Literature-based discovery of confounding in observational clinical data. In AMIA Annual Symposium Proceedings, volume 2016, page 1920. American Medical Informatics Association.

Mallett, R., Hagen-Zanker, J., Slater, R., and Duvendack, M. (2012). The benefits and challenges of using systematic reviews in international development research. Journal of development effectiveness, 4(3):445-455.

Maver, A., Hristovski, D., Rindflesch, T. C., and Peterlin, B. (2013). Integration of data from omic studies with the literature-based discovery towards identification of novel treatments for neovascularization in diabetic retinopathy. BioMed research international, 2013.

Miller, C. M., Rindflesch, T. C., Fiszman, M., Hristovski, D., Shin, D., Rosemblat, G., Zhang, H., and Strohl, K. P. (2012). A closed literature-based discovery technique finds a mechanistic link between hypogonadism and diminished sleep quality in aging men. Sleep, 35(2):279-285.

Mower, J., Subramanian, D., Shang, N., and Cohen, T. (2016). Classification-by-analogy: using vector representations of implicit relationships to identify plausibly causal drug/side-effect relationships. In AMIA Annual Symposium Proceedings, volume 2016, page 1940. American Medical Informatics Association.

Nagarajan, M., Wilkins, A. D., Bachman, B. J., Novikov, I. B., Bao, S., Haas, P. J., Terrón-Díaz, M. E., Bhatia, S., Adikesavan, A. K., Labrie, J. J., Regenbogen, S., Buchovecky, C. M., Pickering, C. R., Kato, L., Lisewski, A. M., Lelescu, A., Zhang, H., Boyer, S., Weber, G., Chen, Y., Donehower, L., Spangler, 
S., and Lichtarge, O. (2015). Predicting future scientific discoveries based on a networked analysis of the past literature. In Proceedings of the 21th ACM SIGKDD International Conference on Knowledge Discovery and Data Mining, KDD '15, pages 2019-2028, New York, NY, USA. ACM.

Özgür, A., Xiang, Z., Radev, D. R., and He, Y. (2010). Literature-Based Discovery of IFN- $\gamma$ and Vaccine-Mediated Gene Interaction Networks. Journal of Biomedicine and Biotechnology, 2010:1-13. Özgür, A., Xiang, Z., Radev, D. R., and He, Y. (2011). Mining of vaccine-associated ifn- $\gamma$ gene interaction networks using the vaccine ontology. In Journal of biomedical semantics, volume 2, page S8. BioMed Central.

Peng, Y., Bonifield, G., and Smalheiser, N. R. (2017). Gaps within the biomedical literature: Initial characterization and assessment of strategies for discovery. Frontiers in research metrics and analytics, 2:3.

Persidis, A., Deftereos, S., and Persidis, A. (2004). Systems literature analysis. Pharmacogenomics, 5(7):943-947.

Petrič, I., Cestnik, B., Lavrač, N., and Urbančič, T. (2012). Outlier detection in cross-context link discovery for creative literature mining. The Computer Journal, 55(1):47-61.

Petric, I., Ligeti, B., Gyorffy, B., and Pongor, S. (2014). Biomedical hypothesis generation by text mining and gene prioritization. Protein and peptide letters, 21(8):847-857.

Petriě, I., Urbaněiě, T., Cestnik, B., and Macedoni-Lukšiě, M. (2009). Literature mining method rajolink for uncovering relations between biomedical concepts. Biomedical Informatics, 42(2):219-227.

Pratt, W. and Yetisgen-Yildiz, M. (2003). LitLinker: capturing connections across the biomedical literature. In Knowledge capture.

Preiss, J. (2014). Seeking Informativeness in Literature Based Discovery. BioNLP.

Preiss, J. and Stevenson, M. (2016). The effect of word sense disambiguation accuracy on literature based discovery. BMC Medical Informatics and Decision Making.

Preiss, J. and Stevenson, M. (2017). Quantifying and filtering knowledge generated by literature based discovery. BMC Bioinformatics, 18.

Preiss, J., Stevenson, M., and Gaizauskas, R. (2015). Exploring relation types for literature-based discovery. Journal of the American Medical Informatics Association, 22(5):987-992.

Pusala, M., Benton, R., Raghavan, V., and Gottumukkala, R. (2017). Supervised approach to rank predicted links using interestingness measures. In BIBM 2017, volume 2017, pages 1085-1092.

Pyysalo, S., Baker, S., Ali, I., Haselwimmer, S., Shah, T., Young, A., Guo, Y., Högberg, J., Stenius, U., Narita, M., and Korhonen, A. (2018). LION LBD: a literature-based discovery system for cancer biology. Bioinformatics, 35(9):1553-1561.

Qi, J. and Ohsawa, Y. (2016). Matrix-like visualization based on topic modeling for discovering connections between disjoint disciplines. Intelligent Decision Technologies, 10(3):273-283.

Qian, Q., Hong, N., and An, X. (2012). Structuring the chinese disjointed literature-based knowledge discovery system. Journal of Information Science, 38(6):532-539.

Ramadan, N. M., Halvorson, H., Vande-Linde, A., Levine, S. R., Helpern, J. A., and Welch, K. M. (1989). Low Brain Magnesium in Migraine. Headache: The Journal of Head and Face Pain.

Rastegar-Mojarad, M., Elayavilli, R. K., Li, D., Prasad, R., and Liu, H. (2015). A new method for prioritizing drug repositioning candidates extracted by literature-based discovery. In BIBM 2015, pages 669-674. IEEE.

Rastegar-Mojarad, M., Elayavilli, R. K., Wang, L., Prasad, R., and Liu, H. (2016). Prioritizing Adverse Drug Reaction and Drug Repositioning Candidates Generated by Literature-Based Discovery. In Proceedings of $B C B$ ' 16 .

Roy, S., Yun, D., Madahian, B., Berry, M. W., Deng, L.-Y., Goldowitz, D., and Homayouni, R. (2017). navigating the functional landscape of transcription factors via non-negative tensor factorization analysis of medline abstracts. Frontiers in bioengineering and biotechnology, 5:48.

Ruthven, I. and Kelly, D. (2011). Interactive information seeking, behaviour and retrieval. facet publishing.

Ryan, P. B., Schuemie, M. J., Welebob, E., Duke, J., Valentine, S., and Hartzema, A. G. (2013). Defining a reference set to support methodological research in drug safety. Drug Safety.

Sang, S., Yang, Z., Li, Z., and Lin, H. (2015). Supervised learning based hypothesis generation from biomedical literature. BioMed research international, 2015.

Santos, B. (2008). Evaluating visualization techniques and tools: What are the main issues. In 2008 AVI 
Workshop on Beyond Time and Errors: BELIV'08.

Schroeder, J., Xu, J., Chen, H., and Chau, M. (2007). Automated criminal link analysis based on domain knowledge. Journal of the American Society for Information Science and Technology, 58(6):842-855.

Sebastian, Y., Siew, E.-G., and Orimaye, S. O. (2015). Predicting future links between disjoint research areas using heterogeneous bibliographic information network. In Pacific-Asia Conference on Knowledge Discovery and Data Mining, pages 610-621. Springer.

Sebastian, Y., Siew, E.-G., and Orimaye, S. O. (2017a). Emerging approaches in literature-based discovery: techniques and performance review. The Knowledge Engineering Review, 32.

Sebastian, Y., Siew, E. G., and Orimaye, S. O. (2017b). Learning the heterogeneous bibliographic information network for literature-based discovery. Knowledge-Based Systems, 115:66-79.

Seki, K. and Mostafa, J. (2009). Discovering implicit associations among critical biological entities. Data mining and bioinformatics, 3(2):105-123.

Shang, N., Xu, H., Rindflesch, T. C., and Cohen, T. (2014). Identifying plausible adverse drug reactions using knowledge extracted from the literature. Journal of biomedical informatics, 52:293-310.

Smalheiser, N. R. (2005). The arrowsmith project: 2005 status report. In International Conference on Discovery Science, pages 26-43. Springer.

Smalheiser, N. R. (2012a). Literature-based discovery: Beyond the abcs. Journal of the American Society for Information Science and Technology, 63(2):218-224.

Smalheiser, N. R. (2012b). Literature-based discovery: Beyond the abcs. Journal of the American Society for Information Science and Technology, 63(2):218-224.

Smalheiser, N. R. (2017). Rediscovering don swanson: The past, present and future of literature-based discovery. Journal of Data and Information Science, 2(4):43-64.

Smalheiser, N. R. and Gomes, O. L. (2015). Mammalian argonaute-dna binding? Biology direct, 10(1):27. Smalheiser, N. R., Shao, W., and Philip, S. Y. (2015). Nuggets: findings shared in multiple clinical case reports. Journal of the Medical Library Association: JMLA, 103(4):171.

Smalheiser, N. R. and Torvik, V. I. (2008). The place of literature-based discovery in contemporary scientific practice. In Literature-based discovery, pages 13-22. Springer.

Song, M., Heo, G. E., and Ding, Y. (2015). Sempathfinder: Semantic path analysis for discovering publicly unknown knowledge. Journal of informetrics, 9(4):686-703.

Spangler, S., Wilkins, A. D., Bachman, B. J., Nagarajan, M., Dayaram, T., Haas, P., Regenbogen, S., Pickering, C. R., Comer, A., Myers, J. N., Stanoi, I., Kato, L., Lelescu, A., Labrie, J. J., Parikh, N., Lisewski, A. M., Donehower, L., Chen, Y., and Lichtarge, O. (2014). Automated hypothesis generation based on mining scientific literature. In Proceedings of the 20th ACM SIGKDD International Conference on Knowledge Discovery and Data Mining, KDD '14, pages 1877-1886, New York, NY, USA. ACM.

Srinivasan, P. (2004). Text Mining: Generating Hypotheses from MEDLINE. Journal of the American Society for Information Science and Technology, 55(5):396-413.

Stegmann, J. and Grohmann, G. (2003). Hypothesis generation guided by co-word clustering. Scientometrics, 56(1):111-135.

Stephanidis, C. (2019). New perspectives into human-computer interaction. User Interfaces for AllConcepts, Methods and Tools, pages 3-20.

$\mathrm{Su}, \mathrm{J}$. and Zhou, C. (2009). Literature-based multidiscipline knowledge discovery: A new application of bibliometrics. Scientometrics \& Informetrics, pages 165-172.

Swanson, D. R. (1986). Fish oil, raynaud's syndrome, and undiscovered public knowledge. Perspectives in biology and medicine, 30(1):7-18.

Swanson, D. R. (1988). Migraine and magnesium: eleven neglected connections. Perspectives in biology and medicine, 31(4):526-557.

Swanson, D. R. (1990). Somatomedin c and arginine: implicit connections between mutually isolated literatures. Perspectives in biology and medicine, 33(2):157-186.

Swanson, D. R. (2011). Literature-based resurrection of neglected medical discoveries. Journal of biomedical discovery and collaboration, 6:34.

Swanson, D. R. and Smalheiser, N. R. (1997). An interactive system for finding complementary literatures: A stimulus to scientific discovery. Artificial Intelligence.

Swanson, D. R., Smalheiser, N. R., and Torvik, V. I. (2006). Ranking indirect connections in literaturebased discovery: The role of medical subject headings. Journal of the American Society for Information Science and Technology, 57(11):1427-1439. 
Symonds, M., Bruza, P., and Sitbon, L. (2014). The efficiency of corpus-based distributional models for literature-based discovery on large data sets. In Australasian Web Conference, pages 49-57. Australian Computer Society, Inc.

Thaicharoen, S., Altman, T., Gardiner, K., and Cios, K. J. (2009). Discovering relational knowledge from two disjoint sets of literatures using inductive logic programming. In IEEE Symposium on CIDM'09, pages 283-290. IEEE.

Torvik, V. I. and Smalheiser, N. R. (2007). A quantitative model for linking two disparate sets of articles in medline. Bioinformatics, 23(13):1658-1665.

Van der Eijk, C. C., Van Mulligen, E. M., Kors, J. A., Mons, B., and Van den Berg, J. (2004). Constructing an Associative Concept Space for Literature-Based Discovery. Journal of the American Society for Information Science and Technology, 55(5):436-444.

Vicente-Gomila, J. M. (2014). The contribution of syntactic-semantic approach to the search for complementary literatures for scientific or technical discovery. Scientometrics, 100(3):659-673.

Vidal, M.-E., Rivera, J.-C., Ibáñez, L.-D., Raschid, L., Palma, G., Rodriguez, H., and Ruckhaus, E. (2014). An authority-flow based ranking approach to discover potential novel associations between linked data. Semantic Web, 5(1):23-46.

Vlietstra, W. J., Zielman, R., van Dongen, R. M., Schultes, E. A., Wiesman, F., Vos, R., van Mulligen, E. M., and Kors, J. A. (2017). Automated extraction of potential migraine biomarkers using a semantic graph. Journal of Biomedical Informatics.

Weidt, F. and Silva, R. (2016). Systematic literature review in computer science-a practical guide. Relatórios Técnicos do DCC/UFJF, 1.

Wilkowski, B., Fiszman, M., Miller, C., Hristovski, D., Arabandi, S., Rosemblat, G., and Rindflesch, T. (2011). Graph-based methods for discovery browsing with semantic predications. In AMIA апnи symp proc, volume 2011, page 1514. American Medical Informatics Association.

Wren, J. D. (2004). Extending the mutual information measure to rank inferred literature relationships. BMC Bioinformatics.

Wren, J. D., Bekeredjian, R., Stewart, J. A., Shohet, R. V., and Garner, H. R. (2004). Knowledge discovery by automated identification and ranking of implicit relationships. Bioinformatics.

Xun, G., Jha, K., Gopalakrishnan, V., Li, Y., and Zhang, A. (2017). Generating medical hypotheses based on evolutionary medical concepts. In IEEE International Conference on Data Mining, volume 2017-November, pages 535-544.

Yang, H. T., Ju, J. H., Wong, Y. T., Shmulevich, I., and Chiang, J. H. (2017). Literature-based discovery of new candidates for drug repurposing. Briefings in Bioinformatics.

Yao, L., Yang, Z., Zhifang, S., and Zhenguo, W. (2008). Research on non-interactive literature-based knowledge discovery. In Computer Science \& Software Engineering, volume 1, pages 747-752. IEEE.

Ye, C., Leng, F., and Guo, X. (2010). Clustering algorithm in literature-based discovery. In Fuzzy Systems and Knowledge Discovery (FSKD), volume 4, pages 1625-1629. IEEE.

Yetisgen-Yildiz, M. and Pratt, W. (2006). Using statistical and knowledge-based approaches for literaturebased discovery. Journal of Biomedical informatics, 39(6):600-611.

Yetisgen-Yildiz, M. and Pratt, W. (2009). A new evaluation methodology for literature-based discovery systems. Journal of Biomedical Informatics. 